\title{
Le réalisme des hypothèses et la Partial Interpretation View $* \dagger$
}

PH. MONGIN, Laboratoire d'Économie Politique, École Normale Supérieure, 45, rue d'Ulm, 75230 Paris CEDEX 05, France

\section{GENERALITES}

Le mot « réalisme » admet, dans la tradition philosophique, tellement d'acceptions différentes que l'on peut douter qu'il y désigne une doctrine, ou même un corps de problèmes, unifié. La discussion prolongée sur le « réalisme scientifique » bénéficie et se ressent tout à la fois de l'indétermination excessive du problème de départ. Comme s'il en était besoin, l'essai mémorable de Milton Friedman, « The Methodology of Positive Economics », est venu compliquer la question d'harmoniques inattendues. Nous nous proposons, après beaucoup d'autres, d'examiner quelques-unes des thèses fondamentales de l'article, celles qui, précisément, se rattachent à la querelle du réalisme, et que l'on peut concentrer dans l'expression anglaise irrealism of assumptions thesis. I s'agira, plus précisément, de comprendre et de critiquer la très célèbre proclamation :

Truly important and significant hypotheses will be found to have 'assumptions' that are wildly inaccurate descriptive representations of reality, and, in general, the more significant the theory, the more unrealistic the assumptions (in this sense). [Friedman 1953, p. 14.]

Pour éviter un premier malentendu, l'auteur ajoutait en substance : l'absence de réalisme est une condition nécessaire, et non pas suffisante, de l'importance de la théorie (ibid., note 12). Muni de cette précision ainsi que d'une distinction proposée par Samuelson (1963, p. 233), nous pouvons isoler, dans la phrase précédente, les deux formes suivantes de la thèse d'irréalisme :

* Reçu 2.7.87

† Initialement présenté au Colloque : « La pensée de K. Popper et la science économique * (Paris, novembre 1986), ce texte a subi de nombreuses modifications, qui reflètent pour partie les remarques utiles de D. Andler, A. Boyer, P. A. Chiappori, P. Engel, G. Granger, P. Jacob, G. Lafleur et P. Salmon. Nous remercions tout particulièrement G. Lafleur et P. Salmon des commentaires écrits qu'ils ont bien voulu nous adresser. 
(1) Si $T$ est une théorie importante et significative de la science empirique, ses hypothèses ne sont pas réalistes (thèse statique ou « F-twist fondamental »).

(2) Plus une théorie $T$ de la science empirique est importante et significative, plus ses hypothèses sont dénuées de réalisme (thèse dynamique ou «F-twist secondaire »).

La réhabilitation paradoxale de l'irréalisme en science prend souvent, dans l'article de 1953, une forme à la fois moins agressive et moins déterminée : « a theory cannot be tested by the 'realism' of its 'assumptions' » (1953, p. 23); « a hypothesis can be tested only by the conformity of its implications or predictions with observable phenomena » (ibid., p. 40).

Les deux phrases qui viennent d'être citées sont, à l'évidence, en retrait par rapport à l'affirmation (1): l'auteur ne veut plus dire que les hypothèses (« assumptions ») doivent être dénuées de réalisme, mais seulement qu'elles peuvent l'être sans qu'il faille voir là un résultat négatif pour la théorie. Par ailleurs, elles tentent une réconciliation singulière de la thèse d'irréalisme avec la méthodologie ordinaire de la science empirique : le test est prohibé-ou plus exactement vidé de toute signification épistémique-pour les hypothèses; il est en revanche admis-et même proclamé nécessaire, cela ressort du contexte-quand on en vient aux conséquences (ou aux « implications», ou aux « prédictions "). Les économistes ont retenu de cette discussion une maxime commode: on ne teste pas une théorie sur ses hypothèses fondamentales, mais sur ses conséquences. Comme notre propos, ici, est d'analyser l'exigence de réalisme en science empirique, nous ne nous attarderons pas sur la distinction-problématique-des assumptions et des predictions, mais nous contenterons d'isoler, dans les phrases immédiatement précédentes, une troisième thèse, qui constitue un affaiblissement de (1):

(3) Si $T$ est une théorie importante et ignificative de la science empirique, ses hypothèses peuvent être ou ne pas être réalistes.

Nous nous proposons d'examiner (1), (2), (3), en faisant largement abstraction du contexte particulier dans lequel ces trois thèses sont apparues. Certes, il sera le plus souvent question, ici, d'économie, car l'enjeu fondamental de (1) ou (3) est qu'elles permettent de neutraliser une objection traditionnellement élevée contre la théorie économique reçue-le manque de réalisme apparent de ses hypothèses fondamentales et, au premier chef, de ses hypothèses psychologiques. Mais l'économie figurera tantôt pour elle-même, tantôt comme un exemple de la science empirique considérée plus généralement : la question du « réalisme des hypothèses » ne lui appartient pas en propre. Par ailleurs, nous 
ne chercherons pas à restituer l'argumentation détaillée de l'article de 1953. Il faut savoir gré à Milton Friedman d'avoir soulevé, dans le style vivant et provocateur où il excelle, une question que la philosophie des sciences répugnait à aborder frontalement; mais on ne doit pas trop attendre du mémorable essai «The Methodology of Positive Economics ». Dans l'abondante littérature qui lui est consacrée, philosophes et économistes se sont livrés à un facile jeu de massacre. La plupart des commentateurs ont conclu que l'article manquait certaines distinctions élémentaires (par exemple, sur le sens des mots « realism ", « assumptions », « predictions ») et interprétait mal les principes canoniques de la philosophie des sciences. Nous ne discuterons pas systématiquement la variante que Friedman a cru pouvoir (1953, p. 20-23) donner de sa thèse (1) en s'aidant de la locution comme si (accepter une théorie $T$, dont les hypothèses fondamentales sont $H$ et les conséquences observables $C$, reviendrait en substance à affirmer « $C$ comme si $H$ »); cette reformulation n'a pas échappé à la critique, loin s'en faut.' II n'y a guère de doute que, si les thèses (1), (2) ou (3) sont d'aventure fondées, ce ne peut être, véritablement, pour les raisons que propose Friedman. C'est pourquoi il vaut mieux discuter ces affirmations en elles-mêmes, sans restreindre a priori le corpus disponible.

Dans ce qui suit, nous considérerons qu'une théorie de la science empirique est donnée sous la forme d'un ensemble de propositions, qui peut être ou bien l'ensemble total de ses conséquences, ou bien un ensemble d'axiomes suffisant pour engendrer l'ensemble précédent. La distinction de ces deux présentations ensemblistes est évidemment utile pour l'analyse des formulations de la thèse sous-jacentes à (3), qui opposent les « hypothèses » et les « implications » d'une même théorie. Il faut toutefois prendre garde que le mot hypothèse peut désigner autre chose que les axiomes de la théorie. Il peut en effet décrire une proposition-axiome ou conséquence-qui intervient dans un explicans déductif-nomologique ou éventuellement statistique. Autrement dit, il peut s'agir d'une loi générale ou d'une condition initiale dans une explication bien formée, sans qu'on veuille indiquer pour autant que l'hypothèse est un axiome. Friedman ne distingue jamais clairement les contextes d'occurrence d'une « hypothèse ». Il ne parvient pas non plus à coordonner les différentes acceptions qu'il envisage pour réalisme. En suivant Nagel (1963), il est facile d'avancer cette première distinction :

1 Une formulation du type * $C$ comme si $H$ * suggère (i) que l'on se désintéresse de la fausseté éventuelle de l'hypothèse $H$; (ii) que l'on a néanmoins produit une dérivation logique correcte de $C$ à partir de $H$. Pour expliciter (i), et peut-être même (ii), il faut pouvoir nommer $H$ : c'est dire que les formulations * $C$ comme si $H *$ ne sont pas du même niveau logique que * si $H$, alors $C$ *; elles appartiennent à la métalangue englobante, et non pas à la langue-objet. Cette difficulté préliminaire affaiblit tous les développements de Vaihinger dans un ouvrage bien oublié aujourd'hui, mais important en son temps, Die Philosophie des Als Ob (1911). 
l'absence de réalisme d'une proposition peut renvoyer simplement à sa fausseté-soit logique, soit factuelle; il peut aussi désigner l'absence de signification empirique immédiate, ce qui est le cas chaque fois que la proposition considérée inclut des termes non directement observables (« force », « utilité », etc.). La distinction très simple de Nagel nous donne la clef la plus utile pour analyser (1) et (2). Par exemple, (1) se subdivise en:

(1.1, interprétation par la fausseté) Si $T$ est une théorie importante et significative de la science empirique, ses hypothèses sont factuellement fausses.

(1.2, interprétation par la théoricité) Si $T$ est une théorie importante et significative de la science empirique, ses hypothèses sont dénuées de signification empirique immédiate.

Lorsqu'elle est prise au pied de la lettre, la variante (1.1) conduit à des difficultés logiques et épistémologiques élémentaires, qui sont liées aux paradoxes de l'implication matérielle. Si l'on sait qu'une proposition scientifique est fausse, on ne doit la faire servir ni à l'explication, ni à la prédiction. La raison en est que, suivant l'analyse ordinaire, ces deux opérations mettent en oeuvre des schémas inférentiels que l'admission de prémisses fausses rendrait trivialement valides : on annulerait ainsi la contrainte, fondamentale dans le modèle déductif-nomologique, d'une dérivation logiquement correcte entre l'explicans et l'explicandum. A quelques exceptions près, les commentateurs de Friedman ont pris acte de ce point : c'est une exigence constitutive de l'explication et de la prédiction bien formées que l'on ne fasse figurer dans l'explicans que des prémisses non réfutées. Nagel (1961, p. 43) l'avait souligné à propos de l'explication. La remarque se transpose d'emblée à la prédiction, soit que l'on invoque la thèse de symétrie, soit que l'on argumente directement : le principe ex falso quidlibet sequitur indique en substance que l'on ne sera jamais à l'abri d'une déception lorsque l'on fait suivre une prédiction d'une prémisse fausse. ${ }^{2}$

(1.1) n'est qu'une variante particulièrement rustique du point de vue instrumentaliste, suivant lequel la vérité des théories se subordonnerait à leur efficacité prédictive. Popper a défini cette tendance comme celle qui fait disparaître la vérité des critères d'appréciation, aussi bien que des objectifs, applicables à la science : les théories ne seraient alors que des « instruments pour prédire les observations» $(1963$, p. 111). On retrouve une expression non triviale de l'instrumentalisme lorsqu'on choisit de reformuler (3), plutôt que (1), en retenant toujours pour

2 Pour le détail de cette argumentation, qui est élémentaire, cf. Mongin 1987, section 2. Elle n'est pas tant dirigée contre Friedman lui-même que contre certains de ses commentateurs, heureusement minoritaires. 
« irréalisme » le sens de « fausseté ». La traduction peut alors déboucher sur l'une ou l'autre de ces variantes:

(3.1 a) Si $T$ est une théorie importante et significative de la science empirique, ses hypothèses sont dénuées de valeur de vérité.

(3.1b) Si $T$ est une théorie importante et significative de la science empirique, nous n'avons pas de raisons de tenir ses hypothèses pour vraies.

Ces deux formulations ${ }^{3}$ sous-entendent que $T$ est importante et significative si et seulement si ses hypothèses rendent comptent adéquatement de ses implications observables connues : l'instrumentaliste tient là sa caractérisation du bon système d'hypothèses, et se refuse, en conséquence, à admettre qu'elles possèdent une valeur de vérité (formulation $3.1 a$, sémantique, de la doctrine) ou que le savant puisse leur en attribuer une (formulation $3.1 \mathrm{~b}$, épistémique, de la doctrine). Cette présentation fait écho aux thèses défendues naguère par Duhem (1906) et, plus récemment, par van Fraassen (1980). ${ }^{4}$ Le progrès par rapport à (1.1) est patent: en suspendant l'attribution de valeur de vérité aux hypothèses, l'instrumentaliste évite de s'embarrasser dans les paradoxes de l'implication matérielle. Il n'en reste pas moins que ces variantes raffinées ont suscité des doutes, pour des raisons proches de celles qui nous ont conduit à rejeter (1.1): quelle est donc la notion de dérivation logique sur laquelle s'appuie l'instrumentaliste, puisqu'il semble vouloir se passer du critère ordinaire de l'inférence correcte (les prémisses transmettent leur vérité aux conclusions, les conclusions transmettent leur fausseté aux prémisses)? ${ }^{5}$

On ne peut faire à Friedman l'injure de lui prêter la thèse (1.1): en dehors du passage singulièrement litigieux sur les formulations en as if, il semble qu'il ne revendique jamais la fausseté des hypothèses d'une théorie scientifique. Mais sa position ne se ramène pas pour autant aux variantes du paragraphe précédent. Elle nous paraît plutôt ressortir à une forme assez inédite d'instrumentalisme, celle qui réinterprète des lois scientifiques réfutées comme vraies dans un certain domaine. Nous

3 Elles coïncident avec des positions classiquement défendues, ou combattues, dans la littérature philosophique sur le réalisme. Pour les retrouver sans emprunter le détour d'une discussion sur la problématique friedmanienne, il suffit d'appliquer le principe de bivalence énoncé par Dummett (1978): une attitude réaliste face à un énoncé se mesure à la capacité de lui attribuer aussi bien la valeur * vrai * que la valeur * faux *. L'instrumentaliste ('antiréaliste) est alors celui qui ne satisfait pas au critère, il revendique ipso facto une thèse apparentée à (3.1a) ou (3.1b).

4 Van Fraassen se réclame de l' $*$ empirisme constructif $*$, mais il y a quelque raison de voir dans sa position (proche du 3.1b) une variante raffinée, épistémique, de l'instrumentalisme.

5 Sur cette difficulté, cf. Jacob 1987, p. 347. Nous avons emprunté à cet auteur la distinction des formulations épistémique et sémantique de l'instrumentalisme. 
discutons en détail cette possibilité, que peu de commentateurs, et, semble-t-il, peu de philosophes des sciences en général, ont choisi d'examiner. ${ }^{6}$ Il est pourtant facile de se convaincre qu'elle représente l'une des options ouvertes à l'instrumentaliste. Celui-ci justifie les hypothèses d'une théorie par la capacité qu'elles ont d'impliquer les énoncés d'observation. Le pouvoir qu'exercent les hypothèses s'apprécie normalement de façon locale : elles permettent de dériver certains énoncés observationnels connus ou visés, typiquement ceux d'un domaine considéré de problèmes ou d'objets matériels. Une hypothèse réfutée peut alors faire l'affaire, pourvu que ses falsificateurs tombent en dehors de cet ensemble, et que l'on puisse ainsi la considérer comme « vraie dans son domaine ». Cette formulation évite à nouveau les difficultés triviales de (1.1). Elle communique avec la seconde caractérisation que Popper fourni de l'instrumentalisme, comme thèse de réduction de la science théorique à la science appliquée. ${ }^{7}$ En effet, c'est en principe la seconde seulement, et non pas la première, qui fait usage d'une notion de « domaine d'application $»$; rendre cette notion disponible à la science théorique aussi, c'est, d'une certaine manière, la ramener à la science appliquée.

La section 2 mobilise la très classique construction du « double langage de la science », que nous précisons ici à l'aide de rudiments de théorie des modèles. Toutefois, ces outils traditionnels révèlent surtout leur efficacité lorsqu'on abandonne la glose de l'irréalisme par la fausseté, et que l'on passe à l'examen de (1.2) et des thèses adjacentes. Dans la variante (1.2), la thèse d'irréalisme ne fait problème, pour les sciences de la nature au moins, qu'aux yeux d'un positivisme dogmatique, et, surtout, de l'opérationnalisme de P. Bridgman. Elle cesse alors d'être spécifiquement instrumentaliste, encore qu'il y ait un lien entre (1.2) et les formulations, que l'on aimerait prêter à Duhem ou à van Fraassen, (3.1a) et (3.1b): afin de refuser d'attribuer une valeur de vérité aux hypothèses d'une théorie non réfutée, l'instrumentaliste peut vouloir tirer argument de la présence, dans ces hypothèses, de termes qui ne reçoivent pas d'interprétation empirique immédiate. Si, dans la sec-

6 Voici le passage témoin qui nous paraît le plus important : * For [a] test to be relevant, the deduced facts must be about the class of phenomena the hypothesis is designed to explain » (1953, p. 13, souligné par nous). Parmi les commentateurs, P. Salmon est l'un des seuls qui aient repéré dans l'Essai le rôle qu'y joue la notion de domaine d'application (1976, p. 781). Le silence des critiques de Friedman, d'ordinaire plus loquaces, s'explique mieux lorsqu'on se reporte à la philosophie des sciences plus généralement : en dehors de l'opérationnalisme de Bridgman, il n'existe pas, à notre connaissance, d'élaboration précise de cette notion cruciale.

7 C. 1963,p. 111. Cette caractérisation paraît logiquement équivalente à la précédente (les théories ne seraient que des * instruments pour prédire les observations ») dès lors que l'on admet que la science appliquée, à la différence de la science théorique, ne se préoccupe que de la prédiction pure (à usage pratique), et non pas aussi de la prédiction de test (à finalité de connaissance). 
tion 3, nous détaillons l'examen de (1.2), c'est pour deux ordres de raisons. D'une part, la polémique contre l'opérationnalisme conserve un intérêt philosophique même si cette doctrine est aujourd'hui abandonnée; en l'occurrence, elle révélera une mise en relation, que nous croyons inédite, entre l'usage des termes théoriques et la recherche de théories réfutables. D'autre part, s'agissant des sciences sociales, la présence de termes non observables dans les hypothèses ne va pas entièrement de soi. Certains auteurs, comme Rosenberg (1976), contestent que la thèse ordinaire de la partial interpretation view se laisse transposer de la physique à l'économie: des termes comme «consommateur », « utilité », « anticipation », ne seraient pas les analogues de « quark» ou « neutrino», parce qu'au sens technique de cette expression, ils sont directement observables. Il faudra tenter d'éclaircir ce point, qui est important pour la thèse de Friedman.

Ces réserves faites, la défense de l'irréalisme est, lorsqu'on vient à l'envisager comme « thèse de théoricité », moins intéressante sous la forme statique (1) que sous la forme dynamique (2). C'est donc sous cet angle que nous la discutons au 4, en même temps qu'une variante qui, chez Friedman, et à un degré moindre, chez Marshall et Machlup, fait système avec elle : le principe d'une liberté sémantique illimitée pour le modélisateur. Dans l'ensemble, nous récusons ces développements de la thèse de théoricité, contrairement au (1.2) que nous admettons, y compris pour les sciences sociales. Les analyses critiques du 4 ne débouchent pas sur une thèse positive que nous puissions mettre au regard de celle de Friedman; mais elles voudraient du moins servir à préciser la problématique difficile du bon usage des termes théoriques en science.

2. LA THÈSE DE FAUSSETÉ A LA LUMIERE DE LA NOTION DE DOMAINE D'APPLICATION

Dans cette section, nous nous proposons essentiellement d'examiner les possibilités qu'offre, pour la thèse de Friedman et la philosophie instrumentaliste en général, une reformulation de prémisses fausses comme des propositions vraies dans un certain domaine. Pour ce faire, nous rappellerons certains aspects de la très classique partial interpretation view of scientific theories. A vrai dire, celle-ci n'est destinée à intervenir complètement qu'au 3; nous n'avons pas besoin, à ce stade, de la thèse d'interprétation partielle, mais seulement de la distinction entre la syntaxe et la sémantique d'un langage scientifique. Le détour par cette construction scolaire ne parait pas seulement utile pour le propos philosophique; il est, secondairement, instructif sur le plan exégétique : contrairement au modèle déductif-nomologique, dont l'Essai de Friedman ne contient aucune occurrence explicite, la conception du « double langage de la science »-cette expression carnapienne semble pour l'instant plus pertinente que celle d' interprétation partielle »y figure 
en termes exprès, accompagnée de modulations qui sont curieuses à relever.

Tout au long de cette section, nous aurons en vue cette situation typique : le scientifique sait que l'une des prémisses de l'explicans-en fait sa loi hypothétique-est fausse; sans défendre rigidement le statu quo, il veut préserver quelque chose de la prémisse réfutée en la restreignant convenablement. Soit l'exemple simpliste: « tous les animaux volent ". Imaginons qu'un naturaliste, ignorant la forme vraie de cette loi (i), explore un territoire $T$, qui est peuplé à la fois d'oiseaux et de lapins. Par hypothèse, il a déjà rencontré des lapins, puisqu'il sait que (i) est fausse. Il veut tenter de reformuler (i) de manière à éviter les implications indésirables. Le risque de cette manoeuvre est patent-la circularité. Définir un domaine d'application par le seul fait que les contreexemples n'y figurent pas est évidemment inadmissible. On peut reprocher à Friedman d'incliner à plusieurs reprises dans cette direction. ${ }^{8}$ La première condition que l'on doive poser sur l'usage d'un concept de domaine est donc l'indépendance épistémique : si $a_{1}, \ldots, a_{n}$ désignent les réfutateurs connus de (i), il faut que la modification évite la forme :

(i i) tous les animaux à l'exception de $a_{1}, \ldots, a_{n}$ volent,

ou toute forme qui lui serait logiquement équivalente. Cela posé, deux possibilités s'ouvrent.

Supposons que l'explorateur ait dans son lexique le prédicat « lapin » et propose la nouvelle régularité :

(i2) tous les animaux à l'exception des lapins volent.

A la différence de la précédente, cette reformulation respecte la condition d'indépendance épistémique; car elle va potentiellement au-delà des données constatées. Par ailleurs, elle fait perdre en réfutabilité-c'està-dire suivant la définition poppérienne, en contenu informatifrelativement à (i). Mais (i2) demeure testable et la perte de contenu reste, intuitivement parlant, dans les limites de l'acceptable. Supposons maintenant que le naturaliste ignore le prédicat « lapin », ou ne veuille pas en faire usage, mais dispose du prédicat « ailé ». La reformulation :

(i3) tous les animaux ailés volent

évite encore la circularité épistémique. Elle est comparable à la précédente en ce sens également qu'elle sauve les phénomènes au prix d'une

8 Methodology of Positive Economics fait de la notion de domaine un usage insuffisamment déterminé. L'exemple le mieux analysé est celui de la loi de la chute des corps (1953, p. 16-19), ce qui n'est pas un hasard (nous aurons à revenir.sur ce point). Les trois exemples qui suivent, dans le passage relatif au « comme si » (p. 19-22), ne s'accompagnent d'aucune délimitation matérielle des domaines, mais seulement de cette indication vague : telle hypothèse * irréaliste " s'applique néanmoins under a wide range of circumstances. Dans ces conditions, on peut craindre que l'assignation de domaine ne coïncide, circulairement, avec une simple exclusion des falsificateurs connus de la loi. 
diminution, intuitivement acceptable, du contenu informatif. Cependant, il y a quelque raison, nous semble-t-il, de ne pas traiter identiquement (i2) et (i3). Il se trouve que (i3) correspond à ce que nous savons être-de notre point de vue d'observateur privilégié-la forme vraie de (i). Cette considération n'a pas à intervenir, puisque nous essayons de restituer la « situation de problème » qui est celle du naturaliste. Mais nous aimerions pouvoir donner un contenu à cette intuition : (i3) semble préférable à (i2), parce qu'elle donne une raison pour éliminer les contre-exemples du champ d'application de la loi. Ce point demanderait à être clarifié; il est sûr qu'il n'a pas la même résonance pour un philosophe instrumentaliste que pour un poppérien.

Une autre démarche, fort différente, consiste à préserver la forme linguistique de (i) et à lui coordonner une règle d'usage. C'est ainsi, précisément, que Friedman explicite son idée de domaine d'application :

We can regard the hypothesis as consisting of two parts: first, a conceptual world or abstract model simpler than the 'real world' and containing only the forces that the hypothesis asserts to be important; second, a set of rules defining the class of phenomena for which the 'model' can be taken to be an adequate representation of the 'real world' and specifying the correspondence between the variables or entities in the model and observable phenomena. [1953, p. 24.]

Dans le cas du naturaliste, une telle règle d'usage paraît difficile à trouver en l'absence d'informations complémentaires sur la « situation de problème ». Nous pouvons toutefois déterminer mieux l'exemple. Supposons qu'un autre explorateur parcoure un autre territoire $T^{\prime}$, sur lequel il n'a rencontré que des oiseaux. Par hypothèse, le premier naturaliste connaît les résultats du second, ainsi que les limites géographiques approximatives de $T$ et $T^{\prime}$. Sa règle d'usage pourrait alors consister à définir $T^{\prime}$ comme le domaine de l'hypothèse (i). Cette adjonction est à certains égards comparable à la reformulation linguistique (i2) : puisque $T$ ne coïncide pas avec l'ensemble des falsificateurs connus de $(i)$, elle respecte la condition d'indépendance épistémique, et elle fait passer d'un énoncé réfuté à un autre qui ne l'est pas, mais qui est moins réfutable. Pour illustrer une règle d'usage qui se comparerait maintenant à (i3), il faut enrichir de nouveau l'exemple. Supposons que $T$ soit composé de forêt et de savane, tandis que $T^{\prime}$ ne comporte qu'une forêt; par hypothèse, le premier naturaliste le sait. Il peut se donner la règle d'usage : (i) ne s'applique qu'aux parties boisées de $T$ et $T^{\prime}$. Ici encore, il faudrait préciser ce que recommande l'intuition : une telle règle paraît plus satisfaisante que la précédente. Elle ne se contente pas de délimiter les cas de test possibles, mais elle amorce une explication de cette délimitation (en un sens qui va au-delà de la rétrodiction correcte des données).

La première et la plus importante conséquence de cette discussion informelle est qu'il existe deux voies pour introduire la notion de 
domaine-l'une est syntaxique et passe par la reformulation de (i), l'autre est sémantique et consiste dans l'adjonction à (i) d'une règle d'usage. Cette distinction essentielle n'échappe pas à Friedman, qui nous semble opter nettement pour la variante sémantique. Il faut maintenant élaborer ce dualisme à l'aide du modèle du « double langage ».

Suivant Carnap (1939 et 1956), une théorie de la science empirique se présente, d'une part, comme un calcul, c'est-à-dire un ensemble de termes, de règles et de propositions primitives, qui livre, en quelque sorte, le squelette logique de la théorie. Elle apparaît, d'autre part, comme un ensemble de règles d'interprétation qui, appliquées à certains des termes du calcul, fournissent la sémantique concrète de la théorie, en rendant possible l'utilisation du calcul à des fins de prédiction ou d'explication.

La théorie comme calcul se compose, plus précisément, de symboles, ou termes primitifs; de règles de formation des formules correctes à partir de ces symboles; de règles d'inférence définies pour les formules correctes; enfin, de formules primitives, normalement distinctes des règles d'inférence. Cette caractérisation très générale s'applique aux calculs logico-mathématiques aussi bien qu'aux calculs non logiquesceux qui correspondent à une théorie de la science empirique, économie, physique, etc. En fait, il faut distinguer, dans les «calculs non logiques », ces deux composantes solidaires: un calcul logicomathématique de base (qui comportera généralement le calcul des prédicats et tel calcul mathématique approprié, par exemple un calcul des nombres réels) et un calcul spécifique, lequel fait intervenir un certain nombre de symboles et de formules ou propositions primitives ne figurant pas dans le calcul de base (mais celui-ci fournit toutes les règles d'inférence). Carnap réserve le terme d' « axiome " aux propositions primitives ajoutées par le calcul spécifique au calcul de base. Ceci est conforme à l'usage courant, puisque l'on désigne ordinairement par "présentation axiomatique » d'une théorie (la mécanique, la microéconomie) non pas le calcul complet qui correspondrait à cette théorie, mais seulement le calcul spécifique : le calcul de base, supposé connu, n'est pas explicité (Carnap 1939, p. 38).

Si la théorie s'identifiait à cette première composante, la signification des termes et des propositions primitives ou dérivées ne renverrait à rien d'extérieur. Ainsi, la signification des termes primitifs coïnciderait avec leur possibilité d'usage correct dans les propositions; inversement, il faudrait regarder les propositions primitives dans leur ensemble comme des énoncés de règles pour l'usage des termes. Il n'y aurait pas lieu de distinguer réellement, dans le calcul, entre les théorèmes qui présupposent le calcul spécifique et ceux qui font intervenir le calcul logicomathématique de base seulement. 
Mais la théorie appartient à la science empirique et il existe une seconde composante qui, justement, lui fournit une sémantique concrète. ${ }^{9}$ Carnap analyse ainsi le rôle des règles d'interprétation: elles consistent à mettre en relation certains des termes primitifs du calcul $C$ avec des énoncés du « langage observationnel $L_{0}(1956, p .47)$. Comme les termes primitifs du calcul spécifique sont reliés les uns aux autres par les axiomes, il est clair, dit Carnap, « que nous n'avons pas besoin d'énoncer une règle sémantique pour chacun d'eux $*(1939, p .62)$ : pour que l'ensemble de la théorie reçoive une interprétation empirique, il suffira qu'un certain nombre d'entre eux, appelés termes observables par opposition aux termes dits théoriques, soient rapportés à des énoncés de $L_{0}$ (le néologisme « observationnel » s'appliquerait plus correctement aux termes que l'adjectif « observable », qui porte normalement sur les choses; mais nous suivrons généralement ici l'usage reçu). De là vient que le modèle carnapien réapparaît généralement dans la littérature contemporaine sous l'étiquette de « partial interpretation view ».

Il nous paraît peu discutable que Friedman adhère-au moins dans le principe-au modèle dualiste de Carnap. Suivant un texte déjà cité (cf. 1953, p. 24), il oppose très explicitement l'aspect de calcul-qu'il assimile plus loin à une « algèbre », ou « logique » (ibid., p. 25)_et l'aspect sémantique (d'ailleurs désigné par l'expression reçue, « règles de correspondance »). Un autre passage soulève quelques problèmes d'interprétation supplémentaires :

Viewed as a language, theory has no substantive content; it is a set of tautologies. Its function is to serve as a filing system for organizing empirical material and facilitating our understanding of it; and the criteria by which it is to be judged are those appropriate to a filing system. ... Viewed as a body of substantive hypotheses, theory is to be judged by its predictive power for the class of phenomena which it is intended to explain. [1953, p. 7-8, souligné par nous.]

Ce texte assez intriguant $n$ 'est pas entièrement passé inaperçu auprès des commentateurs. ${ }^{10}$ Il semble reproduire en substance l'opposition

9 La sémantique d'une théorie de la science empirique désigne ces deux points de vue : d'une part, celui de l'expérimentateur (qui doit traduire en mesures, ou, plus généralement, en observations, certains des concepts de la théorie); d'autre part, celui de l'homme de la rue ou du langage ordinaire (sous ce point de vue, un * atome , par exemple, s'interprète comme un système solaire en miniature). La notion de * langage observationnel », chez Carnap, identifie le point de vue de l'expérimentateur et celui du layman, alors qu'il peut y avoir intérêt à les distinguer.

10 De Alessi (1965) le rapproche du modèle du * double langage » en critiquant, comme nous le faisons dans ce paragraphe, l'assimilation friedmanienne du calcul à un ensemble de tautologies. D'autres auteurs se sont contentés de faire remarquer que Friedman mobilisait une distinction syntaxe-sémantique et que l'Essai reposait le problème, classique en philosophie des sciences, du rôle que jouent les termes théoriques par rapport aux observables : Nagel (1963), Mélitz (1965), Meidinger (1983). En 
carnapienne, tout en y ajoutant une harmonique inattendue : la composante « calcul » pourrait s'analyser comme un « ensemble de tautologies ». Pour apprécier cette idée, il faut sans doute distinguer deux cas : celui où la théorie considérée est logico-mathématique et celui où elle ressortit à une science empirique. Dans le premier cas, l'adjonction de Friedman est admissible. Les propositions primitives du calcul correspondant à une théorie logico-mathématique s'interprètent normalement comme des tautologies; du coup, l'ensemble du système, propositions secondaires comprises, apparaît comme un « ensemble de tautologies ». Mais il n'en va pas de même dans le second cas. Certaines propositions primitives du calcul-les primitives spécifiques ou « axiomes »-appellent normalement une interprétation comme propositions contingentes (susceptibles d'être vraies ou fausses), et non comme tautologies. Maintenir, à ce point, le caractère tautologique du calcul revient très probablement à faire une confusion classique qui mérite d'être exposée au grand jour, car elle empoisonne depuis longtemps la méthodologie économique. ${ }^{11}$ Appelons $P_{1}, P_{2}, \ldots, P_{n}$ les propositions primitives du calcul spécifique et $\mathscr{L}$ l'ensemble des propositions secondaires dérivées des premières. Alors, pour tout $S \in \mathscr{L}$, la formule

$$
P_{1} \& P_{2} \&, \ldots \& P_{n} \rightarrow S
$$

ou, de façon équivalente,

$$
P_{1} \rightarrow\left(P_{2} \rightarrow\left(\ldots\left(P_{n} \rightarrow S\right) \ldots\right)\right)
$$

est une tautologie. Si l'on pouvait remplacer $S$ par $\mathscr{L}$-la « conjonction infinie » de toutes les conséquences des axiomes $P_{1}, \ldots, P_{n}$-on pourrait même dire que le calcul dans son ensemble se télescope dans une unique tautologie. Mais il ne deviendrait pas pour autant un ensemble de tautologies. Car cette dernière formulation imposerait que les propositions de la théorie soient, prises isolément, des tautologies. On a vu que ce n'était pas le cas des $\boldsymbol{P}_{1}, \ldots, \boldsymbol{P}_{n}$; ce n'est pas non plus toujours le cas des $S \in \mathscr{L}^{12}$ Bref, la confusion qui est probablement sous-jacente au passage serait celle-peu flatteuse-de la formule $(F)$ et de ses parties constituantes.

C'est une difficulté plus sérieuse de la conceptualisation friedmanienne qu'elle ne précise pas clairement le statut des énoncés sémantiques. Il est clair qu'ils n'appartiennent pas immédiatement au calcul

revanche, Rosenberg refuse de prêter à Friedman la conception du « double langage * (1976, p. 153-54).

11 Elle apparaît de manière, hẻlas, flagrante chez Hutchison (1938), que Rosenberg (1976) critique avec beaucoup d'à propos. Il est curieux de voir Friedman glisser dans une telle ornière, alors que l'Essai marque-avec les Foundations de Samuelson (1947)_une rupture historique de la méthodologie économique avec les interprétations tautologisantes de la micro-économie.

12 Certaines des conséquences sont évidemment tautologiques. 
formel : dans quel langage sont-ils alors formulés? Ne seraient-ils pas susceptibles, dans un second temps, de s'incorporer au calcul? Carnap, à vrai, dire, n'était guère précis non plus sur le statut de son « langage observationnel » $L_{o}$. La discipline logique qu'on appelle « théorie des modèles » permet dans une certaine mesure de proposer une réponse à ces questions; c'est à l'aide de son formalisme que l'on peut discuter le plus efficacement, croyons-nous, les questions initialement soulevées par Carnap. ${ }^{13}$ En premier lieu, interpréter consiste à mettre en rapport les formules du calcul avec les propriétés concrètes d'ensemblesappelés domaines-sur lesquels les variables de ces formules prennent leur valeur. Plus précisément, si le lexique du calcul $C$ tient dans un ensemble de symboles de variables $\vartheta$ et dans un ensemble de symboles de prédicats $\mathscr{P}$, la théorie des modèles définit une $C$-structure comme un couple $\langle D, P\rangle$, où $D$ est le domaine et $P$ l'ensemble de prédicats symbolisés par $\mathscr{P}$. Une formule de $C$ qui n'est pas une tautologie est vraie dans certaines $C$-structures, appelées modèles, et fausses dans d'autres. En second lieu, la définition d'une $\boldsymbol{C}$-structure ne permet pas de tenir compte aussitôt de la thèse importante de Carnap suivant laquelle tous les prédicats ne sont pas interprétés dans le langage observationnel $L_{o}$. Pour l'intégrer à l'analyse, il faut définir deux étapes sémantiques : la définition d'une $C$-structure a priori, $\langle D, P\rangle$, dans laquelle chaque $p \in \mathscr{P}$ reçoit une interprétation $P^{-} \in P$, et celle d'une $C^{-}$-structure empirique, $\left\langle D, P^{-}\right\rangle$, plus restreinte que la précédente, car seuls certains $p$-les prédicats observables-sont interprétés en $P^{-} \epsilon \mathscr{P}^{-}$. Ce dédoublement de la démarche carnapienne est inévitable étant donné le formalisme de la théorie des modèles; il est aussi souhaitable, car il rend manifeste la distinction, gommée par l'empirisme carnapien, entre interpréter en général (dans une structure qui ne comporte pas que des observables) et interpréter empiriquement (dans une structure qui ne comporte que des observables). En troisième lieu, le statut linguistique des énoncés interprétatifs s'éclaire. On distinguera, à la manière de Tarski, entre une langue-objet, formelle, qui est identique au «calcul » de Carnap, et une métalangue plus riche (elle inclut strictement la première), qui est informelle. Ce que Carnap nomme $L_{o}$ fait partie de la métalangue : ce sont des énoncés informels qui décrivent les propriétés de la $C^{-}$-structure. Les « règles d'interprétation " sont aussi des énoncés métalinguistiques (donc informels), qui mettent en relation les énoncés de la langue-objet èt des énoncés de $L_{o} \cdot{ }^{14}$ Il en va de

13 Nous suivons ici l'approche de Tuomela (1973), qui a fait beaucoup pour clarifier le modèle carnapien et le transformer en une construction logique cohérente. D'autres approches, d'ailleurs également dérivées de la théorie des modèles, paraissent envisageables ici, par exemple celle de Stegmüller (1976) et de l'école structuraliste. Toutefois, le formalisme de Tuomela nous semble rendre compte mieux qu'un autre de la dualité linguistique qu'avait en vue Carnap.

14 Ici également, il serait possible du complexifier le schéma initial, à la façon de Nagel (1961, p. 90) qui distingue trois composantes du langage de la science: les énoncés 
même des « règles de correspondance » dont parle Friedman; sa formulation la plus claire (1953, p. 24, déjà citée) donne à penser qu'elles ne se situent pas au même niveau logique, et ne se formulent probablement pas dans le même langage, que les énoncés du calcul. ${ }^{15}$

Au-delà du commentaire d'un texte assez hermétique, l'outillage conceptuel qui vient d'être introduit permet d'envisager deux approches possibles de la notion de domaine. La première, qui est sémantique, est celle que la théorie des modèles suggère naturellement par ses choix terminologiques : le domaine d'application d'une loi n'est autre que le domaine $D$ sur lequel varient les variables de la formule $F$ correspondant à la loi dans le calcul. L'assignation d'un "domaine d'application * pour une loi se décrit alors comme une restriction imposée sur les interprétations licites du calcul : on se contentera de considérer la collection des $C$-structures de domaine fixé $D$. Il est commode de se représenter chaque $C$-structure comme un monde possible; en se donnant un domaine d'application, le savant détermine la carte des mondes possibles auxquels il entend avoir à faire. Si, comme nous le supposons ici, la loi ( $i$ ) est déjà réfutée, assigner un domaine d'application à (i) revient à décider d'interpréter le calcul dans une collection de $C$-structures qui excluent les falsificateurs connus (par exemple celles dont le domaine $D$ est égal au territoire $T^{\prime}$, etc.). Le point crucial est que cette opération, telle qu'elle vient d'être décrite, ressortit à la métalangue informelle; rien ne dit qu'elle puisse se traduire dans une modification appropriée du formalisme. Ainsi, les navigateurs utilisent une astrophysique insatisfaisante au regard de la théorie physique contemporaine. Ils s'en accommodent parce qu'ils en restreignent l'usage à un domaine convenable-les mouvements des planètes et des étoiles qui sont visibles à l'oeil nu ou à la longue vue. Leur attitude, qui est caractéristique de ce que nous appelons les reformulations sémantiques,

théoriques donnés dans la syntaxe; les * règles de correspondance " entendues strictement, qui mettent en relation dans la syntaxe encore les termes observables et les termes non observables; enfin, les assignations sémantiques informelles, distinguées des précédentes. Cette tripartition, courante dans la philosophie anglo-saxonne des années soixante, ne servirait pas nécessairement notre propos; il n'est pas sûr, d'ailleurs, qu'elle en serve aucun. Hempel (1970) a reconnu en substance qu'il n'y avait pas lieu de distinguer entre les deux premiers types d'énoncés au sein de la syntaxe. La terminologie qu'adopte Friedman est ici malencontreuse, puisque, dans l'usage reçu, les * règles de correspondance * figurent du côté de la syntaxe, et non de la sémantique (note 14); ce point n'a qu'une importance mineure. Il est plus important de souligner que, pour Friedman, l'assignation sémantique fait partie de la science ellemême. Cf. * The important problem in connection with the hypothesis is to specify the circumstances under which the formula works ... such a specification is not one thing and the hypothesis another. The specification is itself an essential part of the hypothesis » (1953, p. 18, souligné par nous). Bridgman (1927) recommandait aux physiciens de développer * l'aspect interprétatif de la physique $n$; on peut dire que Friedman recommande aux économistes de développer l'aspect interprétatif de la théorie économique. 
consiste à préserver le formalisme tel quel et à pratiquer informellement une restriction de domaine. C'est en langue courante, et non pas en langage mathématique, que les officiers de marine pourraient décrire les limites d'application de l'astrophysique traditionnelle. La seconde approche, qui est syntaxique, consiste au contraire à traduire dans le calcul $C$ la restriction que l'on veut imposer à la loi fausse. Le « domaine d'application » apparaîtra alors directement dans l'antécédent de la loi initiale, vraisemblablement par conjonction. Il en va ainsi, par exemple, lorsque la loi de la chute des corps fait place à une formulation plus générale, qui modifie la constance de l'accélération au profit d'une variation expressément spécifiée en fonction de la pression atmosphérique, du volume de l'objet, etc. Cette modification vaut assignation de domaine : elle nous permet de comprendre où et quand la loi originelle de la chute des corps s'applique de manière satisfaisante. Elle a lieu dans la langue-objet : elle est formelle.

Toutes choses égales par ailleurs, la reformulation syntaxique est préférable à la reformulation sémantique. La science empirique cherche certainement à expliciter le plus grand nombre possible de ses résultats dans la langue formelle, dont les enchaînements sont plus faciles à contrôler que ceux de la métalangue. Il existe bien d'autres justifications l'attitude formalisante, mais il n'est sans doute pas nécessaire d'argumenter longuement ici : si le choix se présente dans ces termes, il vaut mieux disposer de la forme explicite non réfutée, « tous les animaux ailés volent » que de l'appréhender indirectement, en combinant la loi, reconnue dans un premier temps comme fausse, "tous les animaux volent ", à une règle d'usage métalinguistique interdisant de l'utiliser en dehors d'un certain domaine $D$. Le vrai problème est que le choix ne se présente pas dans ces termes. Là va apparaître une difficulté majeure pour la position de Friedman et l'instrumentalisme en général. Ces deux points demandent maintenant à être exposés en quelque détail.

C'est un résultat élémentaire, mais fondamental, de la théorie des modèles que toute propriété vraie d'une $C$-structure ne se laisse pas nécessairement exprimer dans une formule de $C$. Ce fait résulte simplement de la différence de complexité de la langue-objet et de la métalangue. Plus formellement, considérons un langage du premier ordre $C$ fixé et une $C$-structure $<D, P>$ a priori quelconque; soit une formule $\varphi$ de $C$ dont les variables libres sont $v_{1}, \ldots, v_{n}$; l'ensemble des $n$-uples $\left\langle a_{1}, \ldots, a_{n}\right\rangle \in D^{n}$ qui satisfont $\varphi$ (la rendent vraie) est dit défini par $\varphi$. Inversement, étant donné un ensemble de $n$-uples $\left\langle a_{1}, \ldots, a_{n}\right\rangle$, on dit qu'il est définissable s'il existe une $\varphi$ de $C$ telle que cet ensemble soit défini. On ne peut espérer que tout sous-ensemble de $D^{n}$ soit définissable : la logique mathématique fournit de nombreux exemples simples d'ensembles (ou de propriétés) qui, pour certains choix de langages égalitaires du premier ordre $L$ et de $L$-structures, ne sont pas définis- 
sables. ${ }^{16}$ Il y a là une dissymétrie remarquable, mais qui n'est pas surprenante à la réflexion : étant donné une formule bien formée, on peut considérer qu'elle vaut « définition implicite » (pour réinterpréter une expression traditionnelle) d'un sous-ensemble, éventuellement vide du domaine, ou plus généralement de $D^{n}$; inversement, le sous-ensemble étant donné, il n'est pas sûr que le langage soit assez riche pour le décrire. En effet, si $D$ est dénombrable, l'ensemble des parties de $D$ est de la puissance du continu, c'est-à-dire qu'il excède l'ensemble total des formules bien formées (qui n'est que dénombrable).

Le très classique problème de la définissabilité a les répercussions suivantes sur l'analyse des domaines d'application : il se peut que l'on ne puisse jamais transcrire dans la langue formelle une restriction métalinguistique parfaitement correcte apportée à une loi fausse. En efet, si D' est le domaine sur lequel on veut désormais faire varier les variables et que $D^{\prime}$ n'est pas définissable, la collection des $C$-structures de domaine $D^{\prime}$ ne se laissera pas décrire formellement; la restriction « $x \in D^{\prime}$ » n'aura pas de traduction dans le langage $C$. On voit maintenant ce qu'il y avait de forcé dans la discussion initiale de l'exemple du naturaliste : comme cet exemple ressortit au langage ordinaire, nous ne pouvions distinguer commodément entre une langue-objet (celle de la syntaxe) et une métalangue (celle de la sémantique). Les contraintes pesant sur le lexique du naturaliste (dispose-t-il du prédicat « lapin »? du prédicat « ailé »?) paraissaient mystérieuses et ad hoc. L'analyse qui précède dissipe ce mystère, en rendant plausible l'existence de contraintes lexicales infranchissables par le scientifique. Si, malgré l'inadéquation de l'exemple initial, on cherchait à lui transposer les conclusions de cette analyse abstraite, on parviendrait sans doute à ceci : le naturaliste ne parviendra peut-être jamais à la formulation correcte " tous les animaux ailés volent ». Cette conclusion demeure, même si, par une hypothèse improbable, le naturaliste savait nommer individuellement chacun des réfutateurs et des falsificateurs de (i).

Le pouvoir de séduction qu'exerce l'instrumentalisme tient en grande partie à l'ignorance des difficultés qui entourent le passage d'une restriction sémantique spontanée à une reformulation syntaxique. Dans un univers panglossien, la première, qui est toujours possible, bénéficierait de la respectabilité scientifique donnée en partage à la seconde. Mais il n'en va pas ainsi, et l'instrumentalisme doit alors ou bien se perdre en n'acceptant comme licites que les reformulations syntaxiques, ou bien rester fidèle à sa propre logique et prêter le flanc à des critiques sévères,

16 Supposons, par exemple, que $L$ soit un langage égalitaire du premier ordre, comportant un symbole de relation binaire $R$ autre que l'égalité, et qu'on l'interprète dans la $L$-structure $\langle Z,\langle>$ ( $Z$ muni de la relation ordinaire d'ordre strict). On montre alors que les seuls sous-ensembles définissables de $Z$ sont $\phi$ et $Z$. Pour une discussion technique de la définissabilité, cf. Kreisel et Krivine, 1967, chap. 6. 
s'il prétend que les restrictions purement sémantiques ont une valeur autre que provisoire. Suivant la première branche de l'alternative, l'instrumentalisme fait place à une autre thèse qui affirme à peu près ceci : lorsqu'une théorie est réfutée, elle disparaît de la science constituée et ne survit plus que dans les limbes de l'heuristique, pour préparer la venue (éventuelle, mais nullement nécessaire) d'une théorie autre, qui tiendra compte de la façon dont s'est produite la réfutation. Suivant la seconde branche de l'alternative, l'instrumentalisme est contraint de réviser le principe, qui semblait tout d'abord s'imposer, de la supériorité des reformulations syntaxiques. On peut envisager des variantes nuancées, et certainement plaidables, d'une telle remise en cause. Il faudrait les examiner en détail. Nous croyons toutefois qu'elles affadiraient à nouveau l'instrumentalisme-pour en faire, par exemple, une épistémologie de l'équilibre temporaire plutôt que des positions normales et durables de la science.

Dans Logic of Modern Physics, Bridgman a cru possible de sauver certaines lois fausses par des reformulations sémantiques : c'est le principe de la « définition opérationnelle ». On dira, par exemple, que les lois de Newton sont vraies lorsque le concept de longueur est interprété au sens du procédé de mesure ordinaire, l'application d'un étalon rigide, etc. Bridgman évite ainsi de reconnaître que les lois de Newton sont fausses lorsqu'on en vient à considérer des corps dotés de très grande vitesse, comme les étoiles ou les particules d'un tube cathodique, puisque le procédé ordinaire ne s'applique plus, justement, à ces corps (il faut alors recourir à des moyens optiques plus raffinés). Cette manière élégante de préserver le contenu de la physique classique n'est pas nécessairement généralisable à d'autres exemples d'histoire des sciences. Il se trouve que la théorie d'Einstein permet de comprendre pourquoi les procédés traditionnels de mesure des longueurs deviennent inadéquats pour les corps dotés d'une grande vitesse : elle prévoit une dépendance explicite de la longueur à l'égard de la vitesse (1927, p. 12). La reformulation sémantique est donc une reformulation syntaxique déguisée. Elle est suggérée par l'existence d'une théorie plus générale, dans laquelle la théorie de Newton s'insère naturellement. Bridgman était évidemment conscient du caractère exceptionnel de son exemple; mais il a paru faire comme s'il était généralisable. En l'absence d'une théorie englobante, comment le savant formalisera-t-il l'existence du domaine (ici, les définitions opérationnelles convenables)? La plupart des exemples de Logic of Modern Physics sont biaisés dans la même direction : Bridgman fait abstraction des contraintes syntaxiques a priori qui pèsent sur le développement de la physique.

Quoiqu'il n'utilise pas cette terminologie, Friedman n'est pas favorable à l'opérationnalisation des concepts micro-économiques; et sa discussion de la loi de la chute des corps (1953, p. 16-19) contredit 
1'hyperempirisme de Bridgman, ou de ses émules en sciences sociales. ${ }^{17}$ Il n'en reste pas moins que friedmaniens et opérationnalistes sont d'accord sur l'intérêt de la notion de domaine, entendue sémantiquement : une théorie peut, et doit lorsqu'elle est fausse, être accompagnée d'une règle d'usage. Et l'on peut élever contre Methodology of Positive Economics l'objection qui vient d'être esquissée contre Logic of Modern Physics. Comme Bridgman avec les lois de Newton, Friedman dispose d'un exemple trop favorable, qui fait écran à une saisie claire du problème linguistique posé par les reformulations. La loi originelle de la chute des corps permet de prédire efficacement le temps de chute « under a wide range of circumstances » $(1953$, p. 18). Nous savons en effet qu'elle convient lorsque le volume du corps n'est pas trop important, etc. Friedman propose en substance de s'en tenir à ces restrictions sémantiques coutumières pour régler l'usage de la loi. Cette position est raisonnable. Mais elle tire sa respectabilité-c'est le point crucial que Friedman néglige de signaler-de la caution que lui apporte la théorie contemporaine des frottements. Autrement dit, nous savons, même vaguement, que les exceptions que nous envisageons ordinairement pour la loi originelle sont prévues par la loi sous sa forme générale: l'accélération dépend en fait du volume du corps, etc. Il s'agit, ici encore, d'une reformulation syntaxique déguisée.

L'exemple des lois de Newton et celui de la chute des corps illustrent le jeu de ce que les philosophes de la physique nomment « principe de correspondance ». Il est trop clair que l'économie ne bénéficie d'aucun de ces exemples privilégiés. Il est possible que les modèles optimisateurs de l'économie néo-classique s'appliquent mieux aux marchés financiers qu'aux autres, mieux aux situations d'offre et de demande qu'aux décisions rationnelles prises hors du marché. Mais cette restriction de domaine informelle n'est cautionnée par aucune métathéorie. Il faut savoir gré à F. Knight d'avoir insisté sur cette règle de politesse épistémologique : on ne peut traiter l'économie comme la physique, en feignant de croire que celle-là disposerait déjà d'une théorie des frottements (cf. 1921 , p. 5). Les arguments logiques de cette section donnent du tranchant à la thèse de Knight : lorsque la théorie des frottements fait défaut, on peut avoir affaire à une situation historique révocable, mais on peut également se trouver prisonnier d'une impossibilité de principe. Il n'est pas exclu que les choix de langage faits initialement par les économistes leur interdisent de passer jamais aux restrictions syntaxiques de domaine.

Il est curieux de noter que Friedman montre à cet égard un pessimisme décapant. Il ne paraît jamais croire que la théorie néo-classique, sous la forme où il la connaît, doive un jour faire place à une théorie plus

17 Nous pensons naturellement aux béhavioristes, dont Bridgman a cautionné l'approche en psychologie. 
générale. Il préfêre la défendre sur la base de son bilan actuel, globalement positif, à ses yeux, parce qu'il existe des domaines d'application favorables. Son hostilité bien connue au formalisme « walrassien ${ }^{18}$ communique sans aucun doute avec cette conception, à la fois apologétique et désabusée, de la discipline telle qu'elle existe. Friedman fait partie de ces auteurs lucides pour lesquels la formulation sémantique d'un domaine d'application ne se transmue pas automatiquement en formulation syntaxique : elle peut désigner une situation permanente.

Notre désaccord avec Friedman ne porte pas sur ce constat, mais sur l'interprétation qu'il appelle en épistémologie normative. Il nous semble peu recommandable de décrire comme « importante et significative " une théorie qui n'est pas parvenue à la reformulation syntaxique. Ou, pour le dire plus précisément, si une pareille théorie doit être caractérisée comme « importante et significative ", ce ne peut être qu'en raison des espoirs qu'elle suscite, et non parce qu'elle ferait déjà partie intégrante de la science. Elle tombe sous le coup des préceptes applicables à l'heuristique des théories, et de ceux-là seulement: au point de vue des règles d'appréciation des théories achevées, elle n'existe même pas. Pour éviter les malentendus que peut susciter une thèse d'apparence aussi brutale, il faut se rappeler ces deux points cruciaux : d'une part, l'appréciation des théories achevées n'est pas le tout du jugement épistémologique (l'heuristique intervient inévitablement); d'autre part, on n'entend pas nécessairement recommander l'abandon d'une théorie lorsqu'on juge négativement ses performances (nous ne voudrions pas exclure, en revanche, que le jugement heuristique puisse déboucher sur une telle extrémité). L'appraisal tel que nous le concevons ici se veut à la fois résolument normatif et dénué de conséquences pratiques immédiates. Ce platonisme assumé n'est un paradoxe que pour des esprits superficiels. De même que l'ordre du droit ne peut se déployer avec cohérence que s'il accepte son idéalité, de même la méthodologie ne peut donner son tranchant à l'appréciation que si elle la détache de la prescription concrète.

Outre la distinction syntaxique/sémantique, d'autres critères interviennent dans le bon usage des domaines d'application. Nous avons évoqué une considération d'indépendance épistémique et une distinction, sans doute assez ténue, entre les restrictions purement négatives et les restrictions déjà explicatives d'un domaine. Nous ne mènerons pas

18 The Marshallian Demand Curve dresse un parallèle célèbre entre Marshall et Walras, à l'avantage du premier (1949, in 1953, p. 89-92). Dans une critique de Lange, Friedman reproche au point de vue * walrassien * de transformer la théorie économique en une classification dénuée d'implications réfutables (1946, in 1953, p. 282-85); à travers Lange, Friedman vise sans doute le programme des Foundations de Samuelson. L'intervention publiée dans Milton Friedman's Monetary Framework reprend le parallèle Marshall-Walras en l'appliquant à Keynes-dont Friedman se dit méthodologiquement proche-et aux keynésiens (1970), p. 18-19 et 145-46). 
ici l'analyse de ces critères intuitifs qu'il faudrait croiser avec la distinction syntaxe/sémantique, mais signalerons seulement une difficulté que l'on rencontre aussitôt qu'on veut les élaborer: le principe de réfutabilité n'aide pas à choisir entre deux restrictions de domaine. Entre (i1), qui ne respecte pas l'indépendance épistémique, et (i2) ou (i3), qui la respectent, le principe imposerait de choisir (i1), la formulation la plus réfutable, mais aussi la moins intéressante. La littérature falsificationniste n'a pas pris conscience de cette difficulté, qu'il faudrait élucider prioritairement si l'on voulait avancer un critère précis du bon usage des domaines.

\section{UNE VERSION MINIMALE DE LA THESE DE THÉORICITÉ}

Dans la variante que nous examinons ici, la thèse (1) se contente d'affirmer que les hypothèses d'une théorie « importante et significative » de la science empirique reçoivent une interprétation empirique seulement indirecte et partielle. Cette sous-détermination des théories par l'empirie tient à la présence dans les hypothèses-ici entendues comme axiomes de la théorie-de termes « théoriques ", c'est-à-dire dont le lien avec les énoncés d'observation est médiat : il transite par le calcul formel et par les règles d'interprétation empirique d'autres termes, les « observables ». La thèse s'identifie donc purement et simplement à la partial interpretation view : sous cette forme, elle n'a, semble-t-il, qu'un seul défaut, celui d'être trop aisément acceptable. Sans doute la philosophie anglo-saxonne a-t-elle, depuis vingt ou trente ans, multiplié les objections contre la partial interpretation view. On a répété à satiété que la notion d'un observable pur était inadmissible, que toute observation était « chargée de théorie ». Mais cette banalité ne peut atteindre que des présentations médiocres ou naïvement positivistes de la thèse, qui, à notre sens, ne prétend pas opérer une fois pour toutes la partition du lexique scientifique en deux groupes de termes. La bonne présentation de la thèse consiste à admettre que la partition est relative à un état donné de la science, voire à une théorie donnée dans un état donné, et même finalement à un point de vue particulier-dicté notamment par telle ou telle expérience-sur une théorie donnée dans un état donné. Cette précision apportée, la banalité de la theory-ladenness indique alors seulement que les observables d'une théorie considérée à un certain point de vue sont des termes théoriques d'une autre théorie, sinon peut-être de cette théorie même lorsqu'elle est prise à un autre point de vue. Suivant une objection plus considérable, ${ }^{19}$ la distinction entre théorique et non théorique ne coïnciderait pas avec celle du non observable et de l'observable: soit que chacune se rapporte à des catégories différentes-grossièrement, les mots pour la première et les choses pour la seconde_, soit que, s'appliquant à une seule et même catégorie, les 19 Cf. van Fraassen 1980, p. 13 sq. 
deux distinctions se chevauchent sans se recouvrir. Pour tenir compte de cette difficulté, nous veillerons à n'appliquer la distinction du théorique et de l'observable qu'aux termes du langage, et non pas à ce qu'ils dénotent. En outre, nous définissons un terme théorique comme un terme non observable (c'est-à-dire dénué d'interprétation empirique immédiate). Une telle manière de voir est conforme à l'approche sémantique que nous adoptons ici pour restituer la partial interpretation view. Elle est, si l'on veut, structurale et formalisante : la partition du lexique en deux semble, jusqu'à un certain point, plus importante que le contenu de chaque composante.

Si, pour les besoins de l'argumentation, on suppose que le sens ultime de " théorique » et d' « observable »n'est pas ce qui fait problème ici, la thèse (1.2) devient presque triviale. La réserve contenue dans le « presque » tient à cette précision : le recours aux termes théoriques est, suivant la formulation conditionnelle de (1.2), une condition nécessaire de la scientificité; tous les philosophes n'admettraient pas l'universalité d'une telle contrainte. Certains auteurs contemporains se retrouveraient plus facilement sur une variante moins ambitieuse: on atteint plus facilement les buts de la science en faisant appel à des théories (i.e. à des énoncés contenant des termes théoriques) qu'en se limitant aux énoncés de lois purement empiriques. Cette conception moins contraignante de la thèse de théoricité est acceptable pour le positivisme logique « libéralisé » qui émerge des écrits de Carnap entre 1935 et 1950, ou de ceux de Hempel et Nagel vers 1950-1960. Il n'est pas sûr qu'il en irait de même pour la thèse (1.2) prise au pied de la lettre. On peut dénier un caractère de nécessité aux termes théoriques en réactualisant cette idée ancienne du positivisme : les termes théoriques n'ont qu'une fonction métalinguistique, ou d'abréviation; ils permettent d'exprimer en langage concis et maniable le savoir accumulé sur les lois empiriques, à l'exclusion de tout autre rôle. Une pareille thèse fait partie intégrante de la critique opérationnaliste des termes théoriques; on la trouve, par exemple, exposée avec de nombreux raffinements formels dans la dernière partie des Models of Discovery (1977) de H. Simon, qui se réclame d'une forme élaborée d'opérationnalisme. Il est probable qu'elle tente encore le Carnap de 1939: celui-ci paraît hésiter entre la thèse ultra-positiviste d'abréviation et l'idée que les termes théoriques sont indispensables (en un sens autre que pragmatique). Bref, malgré sa banalité, la thèse (1.2) n'est pas admise par tous.

Cette discussion préliminaire met déjà en évidence un point important : la thèse de théoricité concerne non pas l'occurrence effective des termes théoriques dans le langage de la science, mais leur éliminabilité. Même lorsqu'elle est abordée suivant le modèle simplificateur et idéalisé du « double langage ", personne n'a jamais prétendu que la science empirique doive comporter des observables seulement. La seule ques- 
tion est de savoir si les termes théoriques ne sont pas éliminables : c'est ainsi que s'exprimerait le fait qu'ils n'ont qu'une fonction d'abréviation. Techniquement parlant, 1'éliminabilité peut s'entendre de manière syntaxique ou sémantique. Nous n'aborderons l'approche syntaxique que dans une variante triviale-l'usage des définitions explicites-délaissant l'approche plus stimulante, mais complexe, de Craig. ${ }^{20}$ Ici comme ailleurs, l'accent de cette étude porte sur la sémantique. Nous donnerons quelques détails, malgré la technicité des développements auxquels on parvient rapidement, sur l'analyse moderne de l'éliminabilité sémantique, dont on fait d'habitude remonter l'idée à Ramsey (1931).

Le philosophe instrumentaliste constituait l'interlocuteur privilégié de la section 2 . Il ne disparaît pas complètement de celle-ci, puisqu'il peut se trouver intéressé à la défense de (1.2): certains auteurs font de l'existence des termes théoriques un argument en faveur de l'instrumentalisme sémantique, celui qui récuse l'attribution de valeur de vérité aux théories (thèse $3.1 a$ ). Une telle inférence est en elle-même sujette à caution. Nous ne la discuterons pas ici pour nous concentrer sur la tâche plus simple d'établir rigoureusement (1.2). Tout au long de cette section, notre véritable interlocuteur polémique sera donc l'opérationnalisme. Nous avions jusqu'à présent décrit cette doctrine comme celle qui veut développer «l'aspect interprétatif de la physique » (Bridgman, 1927, p. vii) et, plus généralement, de la science empirique. On insiste plutôt, d'ordinaire, sur le principe un concept-une opération : «In general, we mean by any concept nothing more than a set of operations; the concept is synonymous with the corresponding set of operations » (ibid., p. 5).

Cette formule est volontiers citée, mais en dehors de son contexte. Voici comment elle se rattache au projet, déjà présenté, d'une formulation explicite des domaines d'application : la définition opérationnelle a pour but de délimiter empiriquement les domaines. Ainsi, la théorie de Newton et celle d'Einstein se distingueront, sémantiquement, par la nature des opérations sous-jacentes à leurs concepts respectifs de longueur. La définition opérationnelle vaut sans doute aussi, chez Bridgman, principe de démarcation : les théories qui ne peuvent y satisfaire-comme la plupart des conceptions psychologiques courantessont réputées dénuées de sens. Mais le plus important est qu'une fois le tri effectué, la synonymie concept-opération impose une classification des ordres de l'expérience qui est elle-même le produit de l'expérience. La phrase la plus importante-mais non la moins obscure-de Logic of Modern Physics est, croyons-nous, celle-ci : « experience is determined only by experience » $(1927$, p. 3$)$.

20 Le théorème de Craig (1953) affirme en substance que, si la « théorie observationnelle "C'est-à-dire l'ensemble des conséquences de la théorie originelle qui ne font intervenir que les prédicats observables-est récursivement énumérable, elle peut être axiomatisée récursivement. On a souvent cru pouvoir conclure de ce résultat que les expressions théoriques sont syntaxiquement éliminables, cf. Tuomela 1973, chap. 3. 
C'est pourquoi notre examen de l'opérationnalisme fera très largement abstraction du sens précis du mot « opération ${ }^{21}$ pour se concentrer sur cette version abstraite du principe bridgmanien : un concept-une expérience. Si l'on se référe à la conception carnapienne du double langage précisée par la théorie des modèles, deux voies s'ouvrent. Ou bien (éliminabilité syntaxique), les termes théoriques sont introduits par voie de définition explicite. Carnap a justement discuté cet aspect de la doctrine opérationnaliste dans Testability and Meaning (1936-37). Ou bien (éliminabilité sémantique), ils figurent dans le calcul de manière éventuellement nécessaire, mais n'apportent rien à l'interprétation empirique, en ce sens que le test des conséquences observables de la théorie est indépendant de leur disparition éventuelle du lexique. Il s'agit là d'une forme beaucoup moins connue d'opérationnalisme, inspirée par le principe de la « Ramsey-élimination ». C'est, nous le montrerons, la seule qui résiste à une critique immédiate.

La première voie consiste à expliciter dans le calcul formel le rôle d'abréviation joué par les termes théoriques. Idéalement, ceux-ci devraient figurer dans des propositions primitives de la forme:

Conc $(x) \leftrightarrow \ldots x \ldots$,

où la sous-formule de droite ne comporte que des prédicats observables, et s'écrit, par exemple, $S(x) \rightarrow R(x)$ (schéma « stimulus-réponse »). En fait, l'opérationnaliste voudra tenir compte du cas plus complexe où les termes théoriques renvoient les uns aux autres. Cette généralisation ne lui coûtera guère s'il impose l'exigence que tous les termes théoriques soient introduits à l'aide du symbole d'équivalence : dans ce cas, on peut évidemment se ramener à $(F 1)$ après un nombre fini de substitutions. La théorie de l'utilité contient de très bon exemples d'éliminabilité par définitions explicites. Dans l'approche moderne, influencée par l'ordinalisme de Pareto, la fonction d'utilité apparaît comme l'indicateur d'un préordre total et continu (« préférence ») sur l'ensemble des choix possibles $X$. Cette interprétation entre dans le calcul formel de la théorie de la manière suivante :

$$
U(x) \geqslant U(y) \leftrightarrow x z_{x} y
$$

le prédicat $z_{x}$ étant par ailleurs défini à l'aide des axiomes ordinaires de complétude, réflexivité, transitivité, continuité, sur l'ensemble $X$. Si l'on pense que, au contraire des valeurs numériques de la fonction d'utilité, les classements de paniers de consommation opérés par le consommateur sont directement observables, la transformation apportée à la théorie est pleinement conforme aux canons de l'opérationnalisme. La théorie des préférences révélées, telle qu'elle est conçue par Samuelson en 1938, fournit un autre cas simple d'éliminabilité syn-

21 Hempel fait de même dans sa réappréciation logique de l'opérationnalisme (1954-1965). 
taxique : le terme $\Theta$, « est révélé préféré à ", y apparaît comme éliminable relativement à quatre autres prédicats observables, ou tout au moins considérés comme tels par Samuelson-« prix », « revenu », « quantité » et « fonction de demande ».22

Ces exemples ont l'intérêt de montrer que les termes théoriques sont quelquefois introduits par définitions explicites dans le calcul formel. Ils ne sont, toutefois, pas généralisables. Carnap (1936-37) a très justement insisté sur le fait que ( $F 1)$ est trop restrictif. Son argument principal, fort simple (1936-37, \$4 et 5), repose sur les paradoxes de l'implication matérielle. Si la définition prenait la forme:

$$
\text { Conc }(x) \leftrightarrow(S(x) \rightarrow R(x)),
$$

il faudrait dire que la sous-formule Conc $(x)$ est vraie quand $S(x)$ est faux, ce qui est manifestement absurde. Le psychologue béhavioriste serait conduit, par exemple, à déclarer « intelligents » non seulement les sujets qui ont passé avec succès un test de Q.I., mais aussi tous ceux qui n'y ont pas été soumis. Pour éviter cette absurdité, il faudrait s'interdire de définir des prédicats tels que l'antécédent $S(x)$ ne soit pas constamment vérifié : cela limiterait drastiquement l'activité scientifique en excluant, par exemple, tous les prédicats introduits sur la base d'une expérience provoquée et tous les prédicats dispositionnels.

Carnap se propose alors de tourner la difficulté en autorisant, à côté des définitions explicites comme $\left(F 1^{\prime}\right)$, d'autres formules d'introduction des prédicats qu'il appelle « énoncés de réduction » :

$$
\begin{aligned}
& S(x) \rightarrow(\operatorname{Conc}(x) \leftrightarrow R(x)) \\
& S(x) \rightarrow(R(x) \rightarrow \operatorname{Conc}(x)) \& S^{\prime}(x) \rightarrow\left(R^{\prime}(x) \rightarrow \neg \operatorname{Conc}(x)\right) \\
& S(x) \rightarrow(R(x) \rightarrow \operatorname{Conc}(x))
\end{aligned}
$$

Les formules $(F 2),(F 3)$ et $(F 4)$ permettent d'éviter les paradoxes douteux auxquels conduisait $\left(F 1^{\prime}\right)$. Si, pour un certain individu $a$, le « stimulus » n'est pas réalisé, on ne conclura ni à Conc $(a)$ ni à $\neg \operatorname{Conc}(a)$. Cette indétermination permet de faire une place aux termes dispositionnels et aux expériences provoquées dans la science empirique, ce qui est le moins qu'on puisse attendre d'une philosophie des sciences?

L'idée carnapienne des « énoncés de réduction » a des conséquences désastreuses sur l'opérationnalisme. Tout d'abord, les termes théoriques ne se laissent plus, en général, éliminer par substitution du definiens au definiendum, comme dans $(F 1)$. D'autre part, la frontière se brouille entre définitions et énoncés empiriques. Si l'on considère $(F 3)$ comme une « définition », elle est vraiment singulière, car elle a des

22 La présentation moderne de l'axiomatique des préférences révelées a d'ailleurs tiré les conséquences de ce fait : le prédicat de Samuelson $\bigotimes$ y est non seulement éliminable, mais éliminé, au profit des quatre prédicats observables. P. ex. Malinvaud 1971, p. 41. 
conséquences empiriques : la conjonction des deux sous-formules fait que l'on ne peut avoir pour un même individu $a: S(a) \& R(a) \& S^{\prime}(a) \&$ $R^{\prime}(a)$. Comme on impose en général aux définitions d'être non créatives, il vaut mieux traiter $(F 3)$ comme un énoncé empirique, même si tous les prédicats qu'il contient ne sont pas directement observables. Mais alors il faut conclure non seulement que les termes théoriques ne sont pas éliminables (au sens syntaxique élémentaire), mais qu'ils jouent un rôle autre que celui, métalinguistique, d'abréviation.

Les « énoncés de réduction » $(F 2),(F 3),(F 4)$, ont également le mérite de faire comprendre pourquoi, en dépit de ce que voudrait l'opérationnalisme, les systèmes formels de la science empirique ne peuvent recevoir qu'une interprétation partielle. Le fait que certains, seulement, des symboles non logiques et non mathématiques du calcul reçoivent une interprétation directe n'aurait pas de conséquences importantes sur la sémantique si les symboles non directement définis étaient toujours introduits sur la base de (F1). Mais ce phénomène devient crucial parce que le système formel va comporter des «énoncés de réduction ». A chaque concept introduit de cette manière va correspondre une zone d'indétermination pour laquelle ce concept ne sera pas empiriquement défini : la solubilité n'a pas de sens empirique lorsqu'on l'applique à des objets qui ne sont pas trempés dans l'eau (Carnap 1936-37, p. 53). De là vient le caractère non seulement indirect, mais partiel, de l'interprétation empirique prêtée aux formules comportant des termes théoriques, et par là au système formel dans son ensemble. A l'acmé de son libéralisme, le Carnap de 1936-37 a vu des avantages épistémologiques, plutôt que des inconvénients, à cette donation partielle de sens empirique : elle préserve en quelque sorte l'avenir, en réservant la possibilité, dans un formalisme ultérieur, d'une réduction de la zone d'indétermination (ibid., p. 59-60).

Nous avons tout naturellement enchainé vers des considérations de type sémantique. Celles-ci constituent le cadre le plus adéquat pour analyser l'opérationnalisme et peuvent d'ailleurs donner matière, dans ce contexte, à des problème techniques encore intéressants pour le logicien contemporain. L'article de Ramsey, Theories (1931), suggère une procédure d'élimination pour les termes théoriques, qui a été identifiée pour la première fois par Braithwaite (1953) et clarifiée bien plus tardivement. Par hypothèse, notre calcul $C$ - un langage du premier ordre-comporte, comme termes non logiques et non mathématiques, un ensemble de prédicats que l'on peut partitionner en deux classes, $\lambda$ et $\mu$, de symboles respectivement « désirables » et * indésirables . Le problème soulevé par Ramsey ne dépend pas essentiellement de la description de ces prédicats comme « théoriques » et « observables "; on demande seulement que l'ensemble total des prédicats se partitionne en deux classes; ce fait indique une nouvelle fois que le contenu de la 
distinction entre «théorique » et « observable " compte moins que l'existence de cette distinction. Supposons que $\mu=\left\{P_{1}, \ldots, P_{n}\right\}$. Etant donné une formule $T$ de $C$, on lui fait correspondre l'énoncé de Ramsey :

$$
T^{R}=\left(\exists \Pi_{i_{1}}\right) \ldots\left(\exists \Pi_{i_{k}}\right) T\left(\Pi_{i_{1}}, \ldots, \Pi_{i_{k}}\right)
$$

que l'on obtient en remplaçant chaque occurrence d'un $P_{i}$ dans $T$ par une variable nouvelle $\Pi_{i_{1}}$ et en quantifiant existentiellement sur cette variable. $T^{R} n^{\prime}$ appartient pas au même calcul $C$ que $T$ : c'est une formule d'un calcul du second ordre, obtenu en enrichissant l'ensemble des variäbles permises dans $C$ d'un ensemble $\left\{\Pi_{i}, \ldots, \Pi_{i_{k}}\right\}$ de variables de prédicats. Il est intuitivement plausible, et démontrable en logique du second ordre, ${ }^{23}$ que $T$ et $T^{R}$ ont les mêmes conséquences dans le vocabulaire restreint aux symboles « désirables » (i.e. l'ensemble des conséquences de $T$ formulées à l'aide des seuls prédicats $\lambda$ est égal à l'ensemble correspondant de conséquences de $T^{R}$ ). Autrement dit, lorsque les $\lambda$ et $\mu$ désignent, respectivement, les symboles de prédicats observables et théoriques, le passage de $T$ à $T^{R}$, ou Ramsey-élimination, garantit que l'ensemble de conséquences observables demeure stable.

L'intuition sous-jacente à la théorie des énoncés de Ramsey est claire : $T^{R}$ dispense de s'engager sur l'existence des prédicats $P_{i_{1}}, \ldots, P_{i_{k}}$ qui figurent dans $T$. Les structures construites pour interpréter $T^{R}$ peuvent apparaître, en un sens, comme plus économiques que les $C$-structures interprétant $T .{ }^{24}$ On a suivi à la lettre la maxime de Russell : « substituer des constructions logiques aux entités inférées ». Mais la substitution entraîne dans une modification du langage initial qui la trivialise: la charge ontologique moindre (si tant est qu'elle soit avérée) se paie d'une prolifération du lexique. La Ramsey-élimination désigne un problème plutôt qu'elle n'offre de solution-outre, naturellement, son caractère abstrait et incommode qui la rend inutilisable pour une réforme concrète de la science. ${ }^{25}$

Certains auteurs ont donc cherché à retrouver autrement le résultat de Ramsey-Braithwaite : la stabilité des conséquences observables lorsque l'on se débarrasse des symboles indésirables $\mu$. Leur approche consiste à appauvrir, plutôt qu'à enrichir, le langage de $C$ - a considérer deux états du lexique, l'état initial, noté $C(\lambda \cup \mu)$, et l'état postérieur à la suppression de $\mu$, noté $C(\mu)$. Cette méthode permet de s'en tenir au premier ordre, ce qui n'a que des avantages techniques. La notion-clef est aiors celle d'appauvrissement (ou d'enrichissement) sémantique.

23 Cf. la proposition de Bohnert dans Tuomela 1973, p. 57.

24 C'est l'argument ordinaire en faveur de la Ramsey-élimination : p. ex. Scheffler 1963 et Hempel 1958.

25 D'autres critiques traditionnelles de la méthode font intervenir deux états successifs d'une même théorie. Lorsqu'une théorie résumée dans un axiome $A$ s'adjoint un nouvel énoncé $B$, faut-il quantifier existentiellement la conjonction $A \& B$, ou bien quantifier sur $A$ et $B$ séparément? 
Soit $T$ une théorie-donnée sous la forme de l'ensemble total des conséquences dans le langage $C(\lambda \cup \mu)$. Cette théorie est interprétée dans des structures $<D, L, M>$-à chaque symbole de $\lambda$ ou $\mu$ correspond un élément de $L$ ou $M$. On peut vouloir transformer une $\langle D, L, M\rangle$ en une structure interprétant des théories $T^{\prime}$, construites dans le langage plus pauvre $C(\lambda)$ : il suffit de faire disparaitre les $M$. On appelle appauvrissement de $\langle D, L, M\rangle$ au langage $C(\lambda)$ la structure $\langle D, L\rangle$ obtenue de cette manière. Inversement, $\langle D, L, M\rangle$ s'appelle enrichissement de $\langle D, L\rangle$ au langage $C(\lambda \cup \mu)$.

Appelons $T(\lambda)$ l'ensemble des conséquences de $T$ formulées à l'aide des seuls prédicats $\lambda$ : c'est ce qu'on peut appeler la « théorie observationnelle » correspondant à $T$. On dira que les symboles $\mu$ sont sémantiquement éliminables de $T$ si l'ensemble des modèles $\langle D, L\rangle$ obtenus par appauvrissement des $\langle D, L, M\rangle$ modèles de $T$ coïncide exactement avec l'ensemble des modèles de $T(\lambda)$.

La question de l'éliminabilité sémantique se schématise donc ainsi :

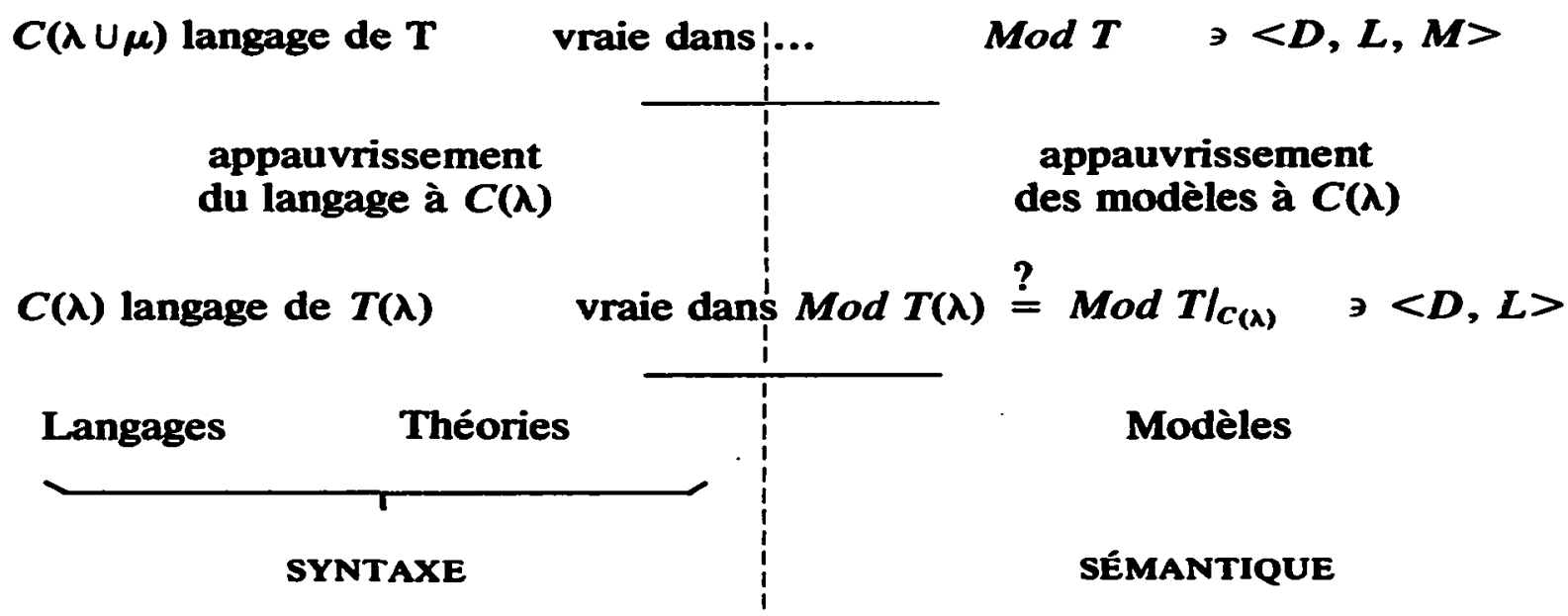

Le symbole $\operatorname{Mod} T$ désigne l'ensemble des modèles de $T ; M o d T / C(\lambda)$ désigne l'ensemble des appauvrissements de ces modèles à $C(\lambda)$. Le formalisme est assez lourd, mais adéquat à l'intuition : si l'on a l'égalité $\operatorname{Mod} T(\lambda)=\operatorname{Mod} T /_{C(\lambda)}$, c'est que les prédicats indésirables n'interviennent pas activement dans la partie de la théorie constituée à l'aide des seuls prédicats désirables; les prédicats théoriques n'influencent pas le test de la théorie observationnelle. On pourra alors déclarer éliminables les prédicats $\mu$, quoiqu'il n'y ait pas lieu de les éliminer effectivement.

Le principal résultat de la théorie de l'éliminabilité sémantique est que celle-ci n'est en général pas possible. Les raisons intuitives de ce résultat négatif méritent d'être exposées, car elles sont philosophiquement interprétables : on va voir qu'on peut les rattacher très directement au projet réfutationniste. La condition $\operatorname{Mod} T(\lambda)=\operatorname{Mod} T /_{C(\lambda)}$ porte sur une double inclusion de classes de modèles. L'inclusion $M o d T / c(\lambda) \subset$ $\operatorname{Mod} T(\lambda)$ est toujours réalisée : si $<D, L, M>$ est un modèle de $T$, son 
appauvrissement à $C(\lambda)$, soit $\langle D, L\rangle$, est-il est facile de s'en convaincre-un modèle de $T(\lambda) .{ }^{26} \mathrm{La}$ condition ne peut manquer d'être vérifiée que parce que l'inclusion réciproque fait défaut: une $C(\lambda)$ théorie $\mathbf{T}^{\prime}$ étant donnée, certains de ses modèles peuvent n'avoir pas d'enrichissements à $\mathrm{C}(\lambda \cup \mu)$ qui soient modèles de $\mathrm{T} .{ }^{27}$ Autrement dit, les langages pauvres donnent lieu à « trop » de modèles; les termes indésirables jouent un rôle pour exclure certains états du monde. Une fois que l'on s'est convaincu de ce point, il fournit un argument réfutationniste en faveur de l'usage des termes théoriques. Il fait aussi apparaître, avec la clarté convenable, le conflit latent qui oppose la doctrine de Popper et celle de Bridgman. En soulignant ainsi le résultat négatif de la théorie de l'éliminabilité sémantique, nous nous situons à l'extrême opposé d'un auteur comme $H$. Simon. Celui-ci se réclame d'une forme sophistiquée d'opérationnalisme en même temps que du réfutationnisme poppérien. Il a voulu réconcilier ses deux inspirations philosophiques en formalisant le principe de réfutabilité de telle manière que celui-ci impliquerait l'éliminabilité sémantique, mais sa tentative rencontre, semble-t-il, des difficultés techniques non négligeables. ${ }^{28}$

Il reste à examiner si la partial interpretation view ne rencontrerait pas des difficultés particulières lorsqu'on tente de la transposer des sciences de la nature-son terrain d'élection-aux sciences sociales. Un auteur, au moins, l'a affirmé de la manière la plus catégorique. Pour A. Rosenberg, la thèse « positiviste » d'interprétation partielle ne s'applique pas à l'économie parce que celle-ci ne se prêterait pas à la distinction préalable des termes théoriques et des termes observables (1976, p. 148-52). Au sens ordinaire du mot, celui qui permet de dire qu'un électron n'est pas observable, il doit se dire des agents économiques: " Economic agents are as observable as human beings are » (ibid.). Quant aux concepts de « raisons ", " croyances », " utilité ", " anticipations ", on peut également prétendre qu'ils sont observables : d'une part, grâce à l'introspection, ils sont accessibles à l'observation d'un individu au moins; d'autre part, si l'on y voit, suivant le point de vue moderne, des termes dispositionnels, en leur attribuant comme référence une tendance à agir, on peut admettre qu'ils sont universellement observables. A ces arguments, Rosenberg ajoute cette considération, qui nous semble en effet importante : les traités d'économie introduisent certains termes fondamentaux de la discipline, comme « agent ", " consommateur », « entrepreneur », sans expliciter, ni paraître supposer, de sens tech-

26 C'est un lemme facile de théorie des modèles, cf. Schoenfield 1967, p. 44.

27 Le contre-exemple que cite Tuomela pour établir ce point $(1973$, p. 61) va encore plus loin qu'il n'est nécessaire. Il montre que, la théorie $T$ étant donnée, il se peut qu'il n'existe aucune $C(\lambda)$-théorie $T^{\prime \prime} \subset T$ dont l'ensemble des modèles coïncide avec $\operatorname{Mod} T / C(\lambda)$ (d'ou, en particulier, $\operatorname{Mod} T(\lambda) \supsetneq \operatorname{Mod} T / C(\lambda)$.

28 Cf. Simon (1977, p. 403-21), précisé dans Simon (1983), et la critique de Rynasiewicz (1983). 
nique particulier, de sorte que la sémantique du langage courant suffirait à les interpréter; or celle-ci les traite peu ou prou comme des observables.

Au travers de cette remarque, on perçoit peut-être une difficulté de la thèse : elle ne distingue pas entre les symboles qui désignent des classes d'individus (" agent ", " consommateur ", « entrepreneur ") et les symboles qui désignent des attributs ou des propriétés de ces individus (" raisons », « utilité », « demande »). On peut facilement admettre que, pour le premier groupe, les économistes s'en remettent à la sémantique du langage ordinaire, et qu'ils en traitent les symboles comme des observables. La situation du deuxième groupe est complexe : « raisons » est un terme du langage courant qui ne doit plus intervenir, en principe, dans l'exposé technique de la théorie; « utilité »s'introduit de manière technique, et nombre d'auteurs insistent sur le fait que ce terme n'est pas observable; " demande » est encore un terme technique, mais que l'on considère normalement comme observable. En bref, le mode d'introduction des termes est plus complexe que ne paraît le dire Rosenberg. Si l'on se réfêre à la pratique des économistes, celle-ci ne peut cautionner le caractère observable des termes que pour certains d'entre eux seulement. La lecture des manuels ou des traités suggérerait que l'on fait généralement passer ainsi la frontière entre la composante observationnelle $T(\lambda)$ de la théorie économique et le reste de cette théorie : les énoncés de $T(\lambda)$ sont typiquement de la forme statique comparative (« si $x$ est un consommateur et que le prix du bien $a$ augmente, $x$ demande plus de $a$ »), tandis que les énoncés de $T / T(\lambda)$ sont typiquement de la forme statique pure ( si $x$ est un consommateur, $x$ maximise son utilité $») .^{29}$

L'analyse qui précède ne vise pas à montrer que la distinction théorique/observable doive s'appliquer à l'économie, mais seulement à rappeler qu'elle intervient effectivement dans la pratique de la discipline. Si l'on en vient maintenant aux raisons que Rosenberg donnait $a$ priori pour disqualifier l'usage de la distinction en science économique, elles appellent certaines réserves. D'une part, pour être véritablement établie, l'observabilité d'un terme doit être universelle. Or l'introspection ne garantit en général qu'une observabilité individuelle. Nous ne voulons pas nier que le positivisme ait tort de dévaluer la méthode empathique, grâce à laquelle certains états mentaux deviennent observables non seulement au sujet qui les ressent, mais à tous ceux qui peuvent les reproduire en eux. Cette méthode n'est sans doute pas

29 Cette partition nous semble première par rapport à celle qu'envisage Rosenberg-entre énoncés micro-économiques et énoncés macro-économiques. Le point du désaccord concerne, ici encore, l'observabilité des prédicats d'appartenance à une classe d'agents. A notre connaissance, les économistes regardent comme faisant partie de $T(\lambda)$ non seulement la loi de demande agrégée, mais aussi la loi de demande microéconomique. 
scientifiquement méprisable. La difficulté de prime abord serait plutôt qu'elle n'opère au mieux que pour certains états mentaux à caractère qualitatif (« disposer d'une utilité accrue »). Il est douteux qu'elle puisse assurer l'observabilité des propriétés numériques (« passer d'une utilité de 2,7 à 7,8 »), sans parler de certaines propriétés qualitatives notoirement embarrassantes à vérifier (« disposer d'une utilité supérieure à celle de l'agent $b$ »). D'autre part, la référence faite au caractère dispositionnel de termes comme « croyances " ou, de nouveau, " utilité », n'emporte pas la conviction. Sans entrer ici dans une querelle délicate, il peut suffire de dire que l'argument est intrinsèquement ambigu : on a pu invoquer la nature dispositionnelle d'un prédicat pour conclure au caractère non observable du terme qui le décrit ${ }^{30}$ En définitive, il semble que les différents arguments de Rosenberg justifient une conclusion plus faible que celle qu'il énonçait : la distinction entre termes théoriques et termes observables ne peut se présenter, en économie, de la même manière qu'en physique. Ceci ne veut pas dire qu'on ne doive pas l'appliquer à l'économie. ${ }^{31}$

\section{VERSIONS FORTES DE LA THĖSE DE THÉORICITÉ}

Si la défense de l'irréalisme se limitait à (1.2)_la thèse initiale de théoricité, elle n'aurait pas suscité tant de résistances chez les épistémologues. En dehors de la problématique de l'éliminabilité, qui présente un intérêt intrinsèque, la simple réitération de la partial interpretation view ne peut passer pour un résultat spectaculaire, fût-ce dans un cas litigieux d'application comme celui de la science économique. On se doute, néanmoins, qu'il y avait là comme un point de passage obligé; l'affrontement de la philosophie des sciences et de l'opérationnalisme représente, certains auteurs, comme Bunge (1973), l'ont bien marqué, le terminus a quo de cette discipline. Dans cette section, nous ferons fonds sur les analyses du 3 pour examiner des variantes plus audacieuses de la thèse de théoricité. L'une d'elles coïncide avec l'expression dynamique de la défense de l'irréalisme (c'est le « $F$-twist secondaire » appliqué au problème des termes théoriques):

(2.2) Plus une théorie $T$ de la science empirique est importante et significative, plus ses hypothèses comportent de termes théoriques.

L'autre cible de la discussion est plus difficile à résumer en une phrase simple, mais elle correspond certainement au projet friedmanien de

30 On peut lier cette conclusion au mode d'introduction retenu pour les prédicats dispositionnels. S'ils s'introduisent par des énoncés de réduction plutôt que par des définitions explicites, il n'est guère plausible de les considérer comme des observables.

31 Cette discussion trop concise ne rend probablement pas justice au point de vue exprimé par Rosenberg en 1976. Nous la poursuivons ailleurs en plus grand détail. 
développer « la partie interprétative de l'économie ». Il s'agit, en substance, de proclamer une liberté sémantique illimitée pour le modélisateur. Lorsqu'on passe de la présentation axiomatique de la théorie à son usage effectif, dans l'explication et le test, la question se pose de savoir si le scientifique hérite d'une sémantique préétablie en même temps que d'un calcul formel. Friedman et, dans une moindre mesure, un autre économiste-philosophe influent et subtil, F. Machlup, suggèrent que ce n'est pas le cas : le scientifique peut recomposer la sémantique de la théorie formelle qu'il utilise au vu de chaque exemple nouveau. Les positions explicites de Friedman et de Machlup sont encore plus ambiguës que cette formulation déjà imprécise en elle-même. Conformément au projet de ce travail, nous ne chercherons pas à justifier en détail l'attribution que nous leur en faisons. Il est donc loisible, pour faciliter la discussion philosophique, de donner une expression plus tranchée au principe de liberté sémantique que nous avons ici en vue:

(4) Lorsqu'il fait usage d'une théorie importante et significative de la science empirique à des fins d'explication ou de prédiction, le savant peut, au vu de l'exemple particulier, modifier la partition des termes en observables et théoriques, remanier les règles d'interprétation déjà employées pour les observables, et plus généralement, ne pas se considérer comme lié par la sémantique qu'il a pu élaborer à l'occasion d'exemples antérieurs.

Pour discuter (2.2) et (4), il faut rappeler quelques évidences fondamentales sur le rôle que l'on peut souhaiter voir jouer aux termes théoriques. En premier lieu, ceux-ci ont une fonction mnémonique, ou récapitulative, qui, nous l'avons dit, est reconnue par les opérationnalistes eux-mêmes. Supposons, par exemple, que l'on analyse la théorie économique comme un ensemble de propositions dont les unes comportent des termes observables $\lambda$ seulement (consommateur, entreprise, bien, marché, prix, quantité) et les autres contiennent, en outre, des termes théoriques $\mu$ (utilité, probabilité subjective, coût d'information). On peut alors se demander si la $C(\lambda)$-expression de la théorie n'est pas préférable à sa $C(\lambda \cup \mu)$-expression, dont le lexique est plus abondant. On répond partiellement à cette question en rappelant que la $C(\lambda)$ expression-la théorie observationnelle-ne se laisse pas nécessairement axiomatiser de manière aussi concise que la $C(\lambda \cup \mu)$-expression : accroître le lexique peut être-est généralement—un moyen de réduire le nombre de formules primitives. Ce fait d'expérience n'est pas en contradiction avec le résultat a priori de Craig. Pour l'économiste, ainsi qu'on l'a souligné à la section 3 , la théorie observationnelle est l'ensemble des régularités de statique comparative, micro-économiques ou agrégées. On voit mal comment axiomatiser cet ensemble de formules 


\section{Ph. Mongin}

sans recourir à l'artifice d'un élargissement du lexique à des termes comme « utilité », " probabilité subjective ", etc.

En second lieu, les termes theoriques aident à concevoir des tests nouveaux. La $C(\lambda)$-expression d'une théorie est, par définition, l'ensemble de ses conséquences empiriques; toutes ne sont pas testables, parce que la forme logique d'une formule peut faire obstacle à sa vérification ou à sa réfutation. Parmi les conséquences testables, on peut vouloir distinguer pragmatiquement, et non plus logiquement, entre celles qui correspondent à des tests répertoriés, peut-être déjà effectués, et les autres. Par exemple, avant le développement de l'école du Public Choice et de la science politique d'orientation néo-classique, le comportement de vote ne constituait pas un test répertorié des hypothèses ordinaires de rationalité individuelle: il n'en va plus de même aujourd'hui, où l'on considère le «paradoxe de l'électeur » comme une anomalie possible de la théorie du choix rationnel. C'est une fonction évidente des termes théoriques, on le voit aussitôt sur cet exemple, qu'ils permettent d'accroître continuellement l'ensemble des tests répertoriés. Une formulation abstraite qui fait intervenir la maximisation de l'utilitéappelle des tests dans les secteurs les plus divers de la vie sociale-le comportement politique aussi bien que la consommation. Il est plus difficile d'imaginer la façon dont un économiste concevrait des tests nouveaux, si son savoir théorique se résumait dans la connaissance de quelques régularités de statique comparative. Sans doute pourrait-il procéder par analogie : la relation entre le prix du beurre et la demande de ce bien est comparable à celle qu'entretiennent la sanction pénale attendue et la « demande de crime », etc. Cette méthode heuristique est parfaitement licite, et d'ailleurs couramment pratiquée par les écoles de sciences sociales qui entendent « généraliser » le modèle économique. L'intérêt des formulations en $C(\lambda \cup \mu)$ est qu'elles permettent de rajouter à celle-ci un autre procédé heuristique plus facilement contrôlable, puisqu'il consiste simplement dans la dérivation logique de conséquences inattendues.

Cette dernière considération ressortit au contexte de découverte. Si l'on s'en tient maintenant au contexte d'appréciation des théories achevées, on rencontre une troisième fonction, qui est peut-être la plus fondamentale : les termes théoriques facilitent l'activité explicative de la science. Au niveau de généralité où il vient d'être exprimé, ce point n'est qu'un truisme; pour voir ce qu'il recouvre exactement, on peut s'arrêter sur un exemple d'explication des lois par les lois: 


\section{(i) Loi $\mathrm{n}^{0} 1-\mathrm{Si} x$ est une entreprise, $x$ maximise ses profits. \\ Proposition explicans \\ (ii) Loi $\mathrm{n}^{0} 2$-Si $x$ est une entreprise et maximise ses profits, $x$ égalise son coût marginal et son revenu marginal. \\ (iii) Loi $n^{0} 3$-Si $x$ est une entreprise et appartient à une branche concurrentielle $y$, son coût mar- ginal est égal à son coût moyen et son revenu marginal est égal au prix qu'elle reçoit. \\ Proposition explicandum \{ \\ (iv) une branche concurrentielle $y$, son coût moyen est égal au prix qu'elle reçoit.}

Cet exemple est représentatif d'une classe très large d'explications scientifiques : étant donné une régularité empirique regardée comme vraie ou suffisamment corroborée, on s'efforcera de la subsumer sous d'autres propositions nomologiques, en respectant les critères habituels du modèle déductif-nomologique, c'est-à-dire, la dérivation logique correcte de l'explicandum, la non-réfutation des prémisses, et leur corroboration indépendante. L'explicandum est « empirique » en ce sens qu'il ne contient que des termes observables ( entreprise », " branche concurrentielle », « coût moyen », « prix reçu », sont ici supposés tels). L'énoncé des trois critères du modèle D.N. n'implique pas, en luimême, que les prémisses de l'explicans doivent comporter des termes théoriques. En toute rigueur, on pourrait prétendre avoir fourni une explication conforme du modèle, si l'on faisait servir la loi empirique no 4 d'explicans à l'énoncé explicandum suivant :

(v) Loi $\mathrm{n}^{0} 5-\mathrm{Si} x$ est une entreprise et appartient à une certaine branche $b$ qui est concurrentielle, son coût moyen est égal au prix qu'elle reçoit.

On voit donc que l'application du modèle D.N. à des explicandums nomologiques ne suffit pas, logiquement parlant, à donner un rôle aux termes théoriques. Ce fait est embarrassant, car il met en lumière une disparité dans la notion ordinaire de théorie. Suivant l'usage reçu, une théorie scientifique désigne un ensemble d'énoncés qui, d'une part, contient des termes théoriques, d'autre part, sert à rendre compte de certaines régularités empiriques bien établies; or ces deux attributs paraissent logiquement indépendants l'un de l'autre. Faute d'une analyse de cette difficulté, on doit s'en tenir à une constatation de fait : les théories que l'on regarde couramment comme explicatives sont toujours dotées d'un vocabulaire théorique. L'exemple qui vient d'être donné 
est, en un sens, archétypique : l'une des prémisses de l'explicans, (i), met en relation un terme observable, « entreprise », avec un prédicat théorique, « maximise ses profits », qui disparaît pendant le cours de la dérivation logique; il en résulte une conclusion, (iv), formulée en termes d'observables seulement.

Une quatrième fonction des termes théoriques, plus difficile à préciser, et plus rarement signalée dans la littérature, met en jeu le principe de réfutabilité. Au 3, nous avons mobilisé des éléments de théorie des modèles pour montrer que l'utilisation d'un lexique enrichi $C(\lambda \cup \mu)$ permettait d'exclure certains états du monde a priori compatibles avec les conséquences $T(\lambda)$ d'une théorie $T$ qui sont formulées dans le lexique pauvre $C(\lambda)$. Pour peu que l'on soit réfutationniste, ce résultat livre un argument de principe en faveur des termes théoriques; paradoxalement, cet argument, qui n'est pas traditionnel, s'est avéré plus facile à présenter rigoureusement que les considérations précédentes sur l'activité explicative de la science. Il a le défaut d'être abstrait et de ne pas assigner clairement une fonction aux termes théoriques. En effet, le formalisme du 3 ne se transpose pas commodément à la « situation de problème » du scientifique. Mais on peut lui trouver un analogue grossier. L'adhésion à une théorie $T$ impose, sur la confrontation de $T$ avec les données, une contrainte qui se traduirait ainsi : le scientifique refusera quelquefois de considérer comme favorables des relevés d'observation (des structures $\langle D, L\rangle$ ) qu'il sait être conformes aux conséquences $T(\lambda)$, mais qui lui semblent constituer la partie observable de mondes possibles (de $\langle D, L, M>$ ) sans rapports avec les propositions les plus théoriques de T. Par exemple, un monétariste ne se satisfera pas de toute espèce de configuration empirique qui comporterait un accroissement de la masse monétaire suivi d'une hausse des prix. Encore faut-il qu'il parvienne à replacer la configuration dans un « scénario » conforme à sa théorie, c'est-à-dire, reposant sur l'existence de courbes d'offre et de demande de monnaie distinctes, stable pour la seconde, etc. Si le monétariste a des raisons de penser que la courbe de demande de monnaie-fonction non observable-a varié pendant la période considérée, il préférera sans doute ne pas prêter valeur de test à la confrontation avec les données. On voit que la procédure logique d'enrichissement des modèles $\langle D, L\rangle$ en $\langle D, L, M\rangle$ n'est pas dénuée de toute analogie concrète; il faudrait, néanmoins, raffiner la comparaison.

On peut lier autrement encore le recours aux termes théoriques et la recherche de théories réfutables. Supposons que, pour une raison quelconque, les termes $\mu$ deviennent observables à l'instar des $\lambda$. Les relevés d'observations prendront désormais la forme $\langle D, L, M\rangle$, au lieu de $\langle D, L\rangle$. Suivant le 3 , les relevés $\langle D, L, M\rangle$ favorables à $T$ donnent naissance, lorsqu'on les appauvrit au vocabulaire observationnel précédent $C(\lambda)$, à des relevés favorables moins nombreux que les 
$\langle D, L>$ en accord avec $T(\lambda)$. En un mot, l'intervention d'observables nouveaux disqualifie certaines observations, formulées dans le vocabulaire ancien, que le savant pouvait regarder comme validantes : la théorie a progressé en réfutabilité toutes choses égales d'ailleurs (à vocabulaire de référence $C(\lambda)$ fixé). Si, derechef, l'on regarde comme un gain le progrès en réfutabilité, on obtient un nouvel argument pour le recours aux termes théoriques: admettre aujourd'hui des termes théoriques dans la science, c'est-puisqu'ils deviendront peut-être observablesfavoriser les réfutations de demain. La transformation de termes théoriques en observables n'est pas un phénomène exceptionnel en histoire des sciences; on cite volontiers, dans ce contexte, les découvertes de la biologie moléculaire qui ont permis de faire évoluer le concept de " gène " vers un statut de quasi-observable.

Au vu de cette énumération sommaire, peut-on prendre position sur les formes extrêmes de la thèse de théoricité? Dans les derniers paragraphes, nous avons anticipé sur la discussion de la thèse dynamique (2.2). Les liens qui s'établissent entre théoricité et réfutabilité constitueraient, pour certains, un argument significatif en sa faveur. On peut y voir-suivant une interprétation généreuse-le véritable contenu que nous livre la synthèse friedmanienne. ${ }^{32}$ Mais il faut ici se méfier d'un malentendu grossier. Etant donné une théorie observationnelle formulée dans un langage $C(\lambda)$, il y a des avantages multiples, que nous venons de développer, à lui préférer son expression théorique, dans le langage plus riche $C(\lambda \cup \mu)$. Mais il n'y a pas de liens logiques entre cette affirmation et celle qui voudrait que l'on préfère à la $C(\lambda \cup \mu)$-expression $T$ une $C\left(\lambda^{1} \cup \mu^{1}\right)$-expression $T^{1}$ vérifiant $\lambda^{1} \cup \mu^{1}=\lambda \cup \mu$ et $\lambda^{1} \varsubsetneqq \lambda, \mu^{1} \supsetneq \mu$, autrement dit une $T^{1}$ réduisant, à lexique inchangé, le nombre des termes observables au profit des théoriques. Si $T^{\mathbf{1}}$, considérée comme ensemble de conséquences, est égale à $T$, la modification sera purement sémantique. On peut conclure sans trop de difficultés qu'elle est régressive. A supposer que le reclassement de certains termes observables comme termes théoriques offre des avantages potentiels-il peut suggérer des tests nouveaux et donner lieu à des explications inédites-il se traduit, sur le champ, par une perte d'information : certains tests déjà effectués deviennent nuls et non avenus, d'autres, qui étaient à venir, deviennent impossibles. Si $T^{1}$ est différente de $T$, la comparaison est infiniment plus délicate et ne se prête pas aux conclusions a priori. Mais il faut au moins

32 Puisqu'il est vrai que Friedman se réclame du falsificationnisme (par ex. 1953, p. 9) et, dans le même temps, exalte le rôle des termes théoriques en science. Cette interprétation vaut évidemment cum grano salis. Etant donné l'usage instrumentaliste que Methodology of Positive Economics fait de la notion de domaine d'application, et la manière très conservatrice dont l'essai organise la défense de la science économique reçue, on ne doit pas prendre exagérément au sérieux l'idée-parfois contenue dans la littérature secondaire-suivant laquelle Friedman serait un * réfutationniste sophistiqué $»$. 
souligner que certains arguments importants en faveur des termes théoriques ne s'appliquent pas à ce contexte. En particulier, il ne peut être question de mobiliser la problématique de l'éliminabilité sémantique et la discussion du paragraphe précédent en faveur de reformulations du type $T^{\mathbf{1}}$.

La théorie économique permet, sur quelques exemples simples, de bien distinguer les cas de figure. Le prédicat " maximise ses profits » est apparu longtemps à la plupart des économistes comme un terme observable. Dans les années trente et quarante, le test de la loi « si $x$ est une entreprise, $x$ maximise ses profits a donné lieu à des interprétations variables, mais beaucoup d'auteurs ont conclu qu'elle était infirmée. L'une des manoeuvres défensives qu'a imaginées Machlup $(1946, p$. 533) revenait simplement à proposer cette redéfinition : les termes « maximise ses profits », « coût marginal », « revenu marginal », renvoient à la perception du chef d'entreprise et ne sont donc pas directement observables. $^{33}$ Telle qu'elle vient d'être présentée, la manoeuvre consîste à remplacer une partition initiale du lexique économique $(\lambda, \mu)$ par une $\left(\lambda^{1}, \mu^{1}\right)$ qui diminue d'un élément le nombre des observables. Ce genre de pratiques est grosièrement ad hoc. Il ne faut pas les rapprocher de celle qui, par exemple, a permis d'unifier les résultats empiriques sur la demande du consommateur autour de quelques axiomes impliquant des termes théoriques comme « maximise son utilité ». Dans ce cas, la démarche consistait à préférer la $C(\lambda \cup \mu)$-expression d'une théorie à sa $C(\lambda)$-expression; elle est fondamentalement progressive.

La distinction qui vient d'être introduite peut servir à clarifier un jugement sévère de Rosenberg sur la partial interpretation view en micro-économie : « Partial interpretation of microeconomic terms insulates the theory in which they figure from difficulties, for it makes determination of the truth of propositions in the higher (more theoretical) reaches of the deductive hierarchy impossible or irrelevant » (1976, p. 143). Cette critique est bienvenue lorsqu'elle porte sur les modifications du lexique dont Machlup donne l'exemple: la partial interpretation view apparaît alors mise au service d'une démarche métathéorique-peu respectable_d'immunisation. Mais elle ne peut convaincre lorsqu'elle vise l'idée même d'interprétation partielle, puisqu'elle tomberait alors sous l'objection générale du 3: l'expression théorique de la micro-économie, comme de tout autre théorie scientifique, est plus riche en réfutateurs que son expression observationnelle. Il est vrai que la méthodologie économique reçue a tout fait pour assimiler les deux points de vue que nous tentons de distinguer ici. Dans un texte curieux, que Rosenberg met en cause à juste titre, Fabian identifie expressément le procédé défensif de Machlup sur la maximisation du

33 Ce n'est pas le seul procédé-loin s'en faut—que les théoriciens néo-classiques aient employé pour défendre l'hypothèse, cf. Mongin 1986. 
profit et la démarche caractéristique de la théorie de l'utilité (1967, p. 63). Il est trop clair que cet auteur a voulu faire bénéficier les ajustements sémantiques ex post, inappréciables pour les théories aux abois, de la respectabilité épistémologique qui s'attache traditionnellement à l'idée de termes théoriques et d'interprétation partielle: on pourrait cesser d'interpréter le terme « maximise ses profits " comme un observable... sous prétexte qu'en science, il existe des termes théoriques et que ceux-ci sont utiles! ${ }^{\text {34 }}$

En définitive, la thèse dynamique de théoricité (2.2) nous semble aussi peu défendable que la thèse statique correspondante (1.2) est banale et, dans l'ensemble, facile à accepter. Au demeurant, elle n'a jamais été défendue sous cette forme précise. Nous l'avons isolée pour la discuter, mais elle est plus concrètement apparue dans le contexte de la méthodologie économique, où elle interfère avec le principe (4), déjà mentionné, de liberté sémantique illimitée du modélisateur. Voici comment ces deux directions s'entremêlent chez Fabian : « Properly selected rules of correspondence can preserve the empirical validity within its proper scope of application » $(1967$, p. 64). Rosenberg remarquait justement que, dans ces lignes, l'attribution de règles sémantiques apparaît comme une question de convenance $(1976$, p. 147). Autrement dit, Fabian se rattache sans équivoque au principe (4). Il en va tout aussi nettement chez son inspirateur, Milton Friedman, comme le montre, mieux encore que Methodology of Positive Economics, le passage d'un article antérieur sur la courbe de demande marshallienne : « The 'commodity' for which a demand curve is drawn is another label, not a word for a physical or technical entity to be defined once and for all independently of the problem at hand » $(1949$, in 1953, p. 57). Ce texte semble impliquer simultanément la dévalorisation du rôle prêté aux observables naturels (le terme « bien », comme celui de « marché » ou de « branche ", pourrait devenir théorique) et le privilège du cas d'espèce dans l'établissement d'une sémantique. C'est ce dernier aspect que nous allons examiner maintenant.

Voici comment l'on peut préciser la formulation (4). L'économiste dispose d'une théorie sous la double forme d'un ensemble suffisant d'axiomes $T$ et d'une classe prédéterminée de structures interprétatives, « naturelles » ou « standards »: pour la théorie de la firme, ce sont les relevés d'observations possibles dans lesquels « firme » s'interprète comme une entreprise au sens juridique du terme, «bien " comme un produit physique, « maximise ses profits », " prix » et « quantité » à l'avenant. Ces conditions de départ étant fixées, le modélisateur peut, suivant le principe (4), se donner un énoncé $F$, pris dans l'ensemble des conséquences de $T$, et redéfinir une classe de structures interprétatives pour $F$ suivant l'opération épistémique-test, explication, 34 Cf. Fabian 1967, p. 64, discuté dans Rosenberg 1976, p. 146. 
prédiction-qu'il veut accomplir sur l'énoncé. Il peut, répétant le procédé de Machlup, faire disparaître toute règle d'interprétation empirique pour le prédicat « maximise ses profits »; il lui est également permis de modifier les règles d'interprétation pour « bien » ou « firme » en rapportant ces termes à des regroupements de produits physiques ou d'entreprises au sens juridique, etc. Le point crucial sur lequel porte (4) est que ces remaniements sont locaux et temporaires. Ils dépendent de $F$ et de l'opération considérée sur cet énoncé. En particulier, rien n'oblige l'économiste à étendre la sémantique nouvelle à l'ensemble de $T:$ il se réserve la possibilité de garder l'interprétation traditionnelle de « bien » lorsqu'il examinera une autre formule $F^{\prime}$. La méthode ici décrite correspond à une pratique traditionnelle de la micro-économie, que l'on peut faire remonter à Marshall (dont se réclame justement Friedman) :

For some purpose it may be best to regard Chinese and Indian teas, or even Souchong and Pekoe teas, as different commodities; and to have a separate demand schedule for each of them. While for other purposes it may be best to group together commodities as distinct as beef and mutton, or even as teas and coffee, and to have a single list to represent the demand for the two combined. $[1920$, p. 100.]

Dans l'usage qui en est fait ici, le schéma carnapien du « double langage » est certainement compatible avec un principe de liberté sémantique; mais non pas, sans doute, avec (4), qui est trop permissif. Un exemple fera voir les dangers de l'absence de toute contrainte. Supposons que l'économiste prétende expliquer la $F$ suivante : « l'industrie américaine de la cigarette n'a pas abaissé sa production lorsqu'on lui a imposé un contrôle des prix ». Supposons aussi, pour les besoins de l'argumentation, que cet énoncé découle facilement de la théorie de la firme en concurrence imparfaite lorsqu'on la conjoint à la condition initiale correspondante, alors qu'on ne peut le rattacher à la théorie concurrentielle. Admettons enfin que l'économiste dispose d'une autre information $F^{\prime}$ : « l'industrie américaine de la cigarette a été soumise à une nouvelle taxe sur les tabacs et l'a répercutée de telle et telle manière sur les prix et les quantités produites », et que $F^{\prime}$, à l'opposé de $F$, soit compatible avec la théorie concurrentielle, mais non pas avec celle de la concurrence imparfaite. Le principe (4) autorise l'économiste à juxtaposer une explication par le monopole dans le premier cas et une explication par la concurrence parfaite dans le second. Pourtant, une telle procédure est contre-intuitive, s'agissant $d^{\prime}$ ' une seule et même industrie dans les deux cas. Si l'économiste adopte le premier explicans, il opte, ce faisant, pour l'interprétation d'après laquelle l'industrie de la cigarette est en situation de concurrence imparfaite. Implicitement, il devrait s'interdire de considérer comme pertinentes des structures dans lesquelles cette industrie serait concurrentielle. Le principe (4) le libère de cette contrainte naturelle: passant de $F$ à $F^{\prime}$, 
l'économiste a le loisir de caractériser à nouveaux frais les structures sémantiques pertinentes.

L'exemple du paragraphe précédent suit approximativement un développement curieux de Friedman (1953, p. 36-37). Celui-ci est, à notre connaissance, le seul auteur à assumer entièrement les conséquences d'une liberté sémantique illimitée pour le modélisateur. Dans ses travaux sur l'entreprise, comme The Political Economy of Monopoly (1952), Machlup ne semblait pas aller aussi loin. Sans doute insistait-il sur la relativité, voire la fragilité, d'une division entre * concurrence parfaite » et * concurrence imparfaite ». Il est loisible, dit-il en substance, d'attribuer ces caractères à des branches qui n'en réunissent pas tous les attributs; le choix de l'un plutôt que l'autre dépendra souvent du type de problème traité. Mais Machlup ne va pas jusqu'à permettre de traiter une seule et même entité économique suivant deux sémantiques contradictoires. La citation de Marshall paraît également en retrait par rapport à l'extrémisme friedmanien. Pour en retrouver l'équivalent, il faudrait imaginer cet exemple contourné : l'économiste peut, s'agissant du même consommateur, étudier l'élasticité de substitution du thé au café en identifiant les qualités Souchong et Pekoe, et celle du thé à la tisane en les distinguant. Il n'est pas nécessaire de détailler longuement les inconvénients méthodologiques de la conception friedmanienne de la sémantique. Dans le cas d'espèce, le théoricien de l'entreprise se condamne à ne rien expliquer du comportement de l'industrie américaine de la cigarette. Comme les bons auteurs y ont justement insisté, un explicans ne remplit pas sa fonction s'il n'a d'autre vertu que de rendre possible la dérivation logiquement correcte de l'explicandum; encore faut-il qu'il soit testé indépendamment de celui-ci. (4) vide cette exigence traditionnelle de tout contenu. On pourra toujours disqualifier un réfutateur de l'explicans en redéfinissant la classe des structures interprétatives pertinentes. C'est précisément ce qui arrive dans l'exemple précédent, puisque $(F)$ réfute l'explicans hypothétique de $\left(F^{\prime}\right)$, et réciproquement. Il faut remarquer que cette critique est indépendante de la caractérisation, théorique ou observationnelle, que l'économiste friedmanien aurait pu retenir pour les termes de « concurrence parfaite * et

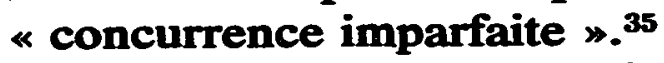

Comment restreindre le jeu du principe de liberté sémantique du modélisateur? Deux considérations s'imposent à l'attention. D'une part, les assignations sémantiques doivent être au moins localement cohérentes. Le micro-économiste peut interpréter la demande de thé au sens d'une qualité particulière s'il veut expliquer l'évolution de la consommation des boissons, et au sein d'un large regroupement de ces qualités s'il construit un modèle d'équilibre général. Mais l'examen de chaque cas doit respecter la contrainte générale: on ne soumet pas

35 Elle concerne seulement la cohérence dans le choix des structures, que celles-ci soient de la forme $<D, L>$ ou $<D, L, M>$. 
l'ensemble des structures interprétatives à des restrictions que la syntaxe de la théorie déclare contradictoires. Au-delà de la polémique contre le friedmanisme, on peut s'étonner de la fréquence avec laquelle cette condition apparaît violée dans la modélisation économique. ${ }^{36}$

D'autre part, le principe qui réglait l'usage des domaines d'application trouve à s'appliquer de nouveau : le scientifique doit s'efforcer de transformer syntaxiquement les ajustements sémantiques imprévus. Marshall a raison de penser que la loi de demande ne s'interprète pas toujours à partir de la notion physique ou commerciale de « bien ». Mais il a tort de réduire le choix du niveau d'agrégation pertinent à une décision métalinguistique. Suivant la reconstruction individualisterationaliste de la loi de demande que poursuit la théorie microéconomique, le découpage technique de la notion de «bien » résulte des distinctions subjectivement pertinentes pour les décideurs, en l'occurrence du contenu de chaque bien concret en caractéristiques, ainsi que des élasticités de substitution entre ces caractéristiques. Le choix du niveau d'agrégation, pour le test de la loi, ne se présente donc pas seulement comme celui d'une classe de structures interprétatives. Il a virtuellement la forme d'une hypothèse auxiliaire que l'économiste pourrait incorporer à la syntaxe. Lorsqu'elle est possible, ce qui est le cas ici, la reformulation syntaxique révèle une nouvelle fois sa supériorité relativement aux assignations sémantiques pures : elle permet d'accroître le nombre des tests en révélant le caractère d'hypothèse de ce qui, chez Marshall, a le caractère inattaquable d'une interprétation. On pourrait analyser de même la modification que Machlup proposait du prédicat « maximise ses profits ». Difficilement acceptable telle quelle, cette révision sémantique l'est devenue lorsque s'est développée une théorie en bonne et due forme des profits attendus-avec la construction, dans les années 1945-1955, de l'hypothèse de l'utilité espérée. A ce stade, l'idée suivant laquelle les profits sont des « grandeurs psychiques » cesse d'être intempestive. Le principe régulateur, suivant lequel il faut rechercher la contrainte syntaxique maximale, a rempli son office. ${ }^{37}$

36 L'auteur a eu entre les mains une thèse de macro-économie dont les résultats étaient testés sur données trimestrielles reconstruites et dont l'une des hypothèses-celle des " anticipations rationnelles "-stipulait la coïncidence entre l'information des agents et celle du modélisateur, alors même que, sur la période considérée, la seule information disponible était semestrielle. On pourrait multiplier ce genre d'exemples incongrus.

37 Derechef, il serait possible d'enchaîner l'examen critique de la modélisation économique sur celui des thèses méthodologiques. Les économistes font quelquefois jouer avec rigueur l'exigence syntaxique, comme on l'a rappelé à propos de la théorie de l'utilité espérée. Mais, comme Favereau (1983) l'a montré sur l'exemple des théories néo-classiques du marché du travail, il n'est pas rare qu'ils projettent sur un formalisme malingre une sémantique surabondante et impiécise. De tels travaux, friedmaniens dans la méthode, ne débouchent jamais sur la reformulation syntaxique. 
Nous avons introduit dans la discussion, sans le justifier, le terme nouveau de modélisateur. Il se trouve que les thèses élaborées par Marshall, Machlup ou Friedman, sinon la méthodologie économique en général, privilégient une conception de la science par « modèles " plutôt que par « systèmes »: ${ }^{38}$ elles insistent sur l'individualité des cas, en regardant les théories comme des répertoires souples et incomplets, et non pas comme des moyens de classement. Il n'est pas question, ici, de prendre position contre l'approche par « modèles », qui a des justifications particulières dans les sciences sociales, mais aussi de très respectables antécédents parmi les sciences de la nature. ${ }^{39}$ II semble en effet inévitable que les difficultés liées à l'appréhension de l'individuel interfêrent avec l'établissement d'une sémantique concrète pour une théorie : ce ne peut être un hasard que ces deux déterminations coexistent, justement, dans le halo de sens du mot « modèle ». Autrement dit, on ne peut prétendre statuer sur la sémantique des théories scientifiques sans réserver une large place à la variabilité des assignations. Mais le principe (4) nous semble caricaturer cette exigence en refusant de la déterminer. La méthode des modèles, inévitable, comporte le risque évident de transformer la science en une multitude de résultats partiels, dont la mise en rapport logique ou épistémique devient de plus en plus difficile. Pour ne pas trahir complètement l'individualité d'une situation, les scientifiques doivent, à la fois, ajuster la sémantique de leur théorie et en surdéterminer la syntaxe par des hypothèses auxiliaires. Ces deux mouvements ne sont pas toujours faciles à distinguer, parce que les hypothèses auxiliaires ne se laissent pas toujours formaliser au même degré que le corps de la théorie et apparaissent alors prises en compte dans la sémantique de la théorie plutôt que dans son calcul. Il est aussi vrai que la modélisation-par contraste avec l'axiomatisation-n'impose pas d'expliciter toutes les prémisses qui sont logiquement nécessaires à une dérivation correcte des conséquences: par exemple, les économistes se sont contentés, pendant très longtemps, de modéliser l'équilibre de marché sans expliciter les hypothèses d'anticipations dont ils avaient besoin pour inférer un comportement d'offre ou de demande. Ainsi, les spécifications du corpus théorique au cas par cas, et le maintien d'un non-dit dans chaque cas, contribuent puissamment à l'incommensurabilité des modèles. Il faut veiller à ne pas aggraver cette situation, qui est en quelque sorte normale, par une trop grande permissivité sémantique.

\section{CONCLUSION EN FORME DE RÉSUMÉ}

Cette étude portait sur la question-activement débattue depuis M. Friedman-du réalisme des hypothèses en science empirique, et plus

38 Au sens de Pariente 1973, chap. 7.

39 Voir p. ex. Bachelard (1979). 
particulièrement en théorie économique. On peut en schématiser les résultats en prenant l'exemple des propositions nomologiques fondamentales de la micro-économie, « si $x$ est une entreprise, $x$ maximise ses profits », ou bien : « si $x$ est un consommateur, $x$ maximise son utilité ». Quelle signification faut-il donner à la thèse d'après laquelle ces propositions seraient importantes scientifiquement quoiqu'elles soient éventuellement dénuées de réalisme? Suivant une première analyse, la plus simple, de l'irréalisme, elles sont scientifiquement importantes quoique fausses. Comme une telle position est insoutenable (1), il faut la reformuler, par exemple de cette manière: les propositions nomologiques fondamentales de l'économie seraient vraies dans leur domaine d'application. On a identifié l'instrumentalisme de Friedman à cette variante particulière que la philosophie des sciences n'a guère discutée jusqu'à présent. On a montré que la reformulation d'une loi réfutée comme loi vraie dans un certain domaine doit satisfaire à certains critères épistémiques intuitifs, et surtout, tendre vers un remaniement de la syntaxe, et non pas seulement de la sémantique. Réalisées en physique dans certains cas exceptionnels ( principe de correspondance »), ces conditions ne le sont pas en économie; il paraît donc peu recommandable de chercher à sauver les hypothèses fondamentales de cette discipline en recourant à une idée de domaine d'application (2). Une variante plus adéquate consiste à interpréter l'absence de réalisme comme théoricité, c'est-à-dire à déclarer que les prédicats « maximise ses profits », « maximise son utilité », sont non observables. Cette option revient, au minimum, à réitérer la célèbre partial interpretation view of scientific theories. Elle peut convenir à l'instrumentalisme : suivant une inférence problématique, que l'on n'a pas examinée ici, les partisans de cette doctrine ont quelquefois voulu conclure de la présence de termes théoriques à l'impossibilité d'assigner une valeur de vérité aux énoncés qui les contiennent. Mais la partial interpretation view peut aussi bien satisfaire le philosophe réfutationniste : on a montré que la recherche de théories réfutables constituait un argument frappant, quoique peu connu, en faveur des termes théoriques. Leur rôle n'est contesté globalement que par l'opérationnalisme : au vu d'une double analyse, syntaxique et sémantique, de l'éliminabilité, les thèses de cette école ne semblent guère tenables. On a également discuté brièvement, pour conforter la partial interpretation view, des difficultés particulières que, d'après certains auteurs, la science héorique susciterait pour la distinction entre termes théoriques et observables (3). A côté de l'interprétation minimale de la théoricité, qui consiste simplement à rappeler le rôle fondamental du premier groupe de termes, on peut envisager la conception qui, chez Friedman, et dans une moindre mesure, chez Marshall et Machlup, domine l'approche de la firme : il y aurait avantage à multiplier les termes théoriques aux dépens des observables et à laisser le mo- 
délisateur recomposer sur chaque cas particulier la sémantique des hypothèses économiques. Ces deux affirmations ne semblent pas dépendre d'une adhésion préalable à l'instrumentalisme; elles formalisent plus ou moins une pratique spontanée des économistes. La première est une variante dynamique de la thèse de théoricité, que Machlup illustre bien en théorie de la firme. Il faut soigneusement la distinguer de l'affirmation statique correspondante, laquelle s'appliquerait, en revanche, à la théorie du consommateur, et qui indique en substance que l'expression théorique d'une hypothèse est préférable à son expression observationnelle. La variante dynamique n'est pas tenable : elle revient à une défense ad hoc de la micro-économie traditionnelle. La seconde affirmation proclame la liberté sémantique illimitée du modélisateur: elle s'avère épistémologiquement dangereuse parce qu'elle fait obstacle au rôle du test indépendant, c'est-à-dire au bon fonctionnement de l'explication scientifique. De façon générale, elle favorise la balkanisation des disciplines, en exagérant la tendance naturelle de la modélisation, aux dépens des démarches unifiantes de la science comme l'axiomatisation, et il faut lui opposer le principe d'une liberté sémantique réglée du modélisateur (4).

\section{BIBLIOGRAPHIE}

Alessi (de), L. (1965) * Economic Theory as a Language *, Quarterly Journal of Economics, 79, $472-77$.

Bachelard, S. (1979) * Quelques aspects historiques des notions de modèle et de justification des modèles $\%$, in P. Delattre et M. Thellier, Elaboration et justification des modèles, t. 1. Paris, p. 9-20.

Braithwaite, R. B. (1953) Scientific Explanation. Cambridge.

Bridgman, P. (1927) The Logic of Modern Physics. New York.

Bunge, M. (1973) Philosophy of Physics. Dordrecht.

Carnap, R. (1936-1937) * Testability and Meaning *, Philosophy of Science, 3 (1936), et 4 (1937), réed. in H. Feigl et M. Brodbeck, Readings in the Philosophy of Science. New York 1953, p. 47-92.

(1939) * Foundations of Logic and Mathematics *, in O. Neurath, R. Carnap et C. Morris, Foundations of Unified Science, vol. 1. Chicago 1938-1972, p. 139-214 (1ère éd. 1939).

(1956) * The Methodological Character of Theoretical Concepts *, in H. Feigl et M. Scriven, Minnesota Studies in the Philosophy of Science, t. 1. Minneapolis.

Craig, W. (1953) * On Axiomatizability Within a System *, Journal of Symbolic Logic, 18 , 30-32.

Duhem, P. (1906) La théorie physique, son objet, sa structure, 2e éd. 1914. Rééd. Paris 1981. Dummett, M. (1978) Truth and Other Enigmas. Cambridge, Mass.

Fabian, R. G. (1967) * An Empirical Principle for Deductive Economics *, Southern Economic Journal, 34, 53-66.

Favereau, O. (1983) * Le chômage est-il volontaire? $»$, in C. Meidinger, La nouvelle économie libérale, chap. 2. Paris.

van Fraassen, B. C. (1980) The Scientific Image. Oxford.

Friedman, M. (1946) * Lange on Price Flexibility and Employment: A Methodological Criticism ", American Economic Review, 36, 613-31 (repris in M. Friedman, Essays in Positive Economics, 1953). 
(1949) * The Marshallian Demand Curve *, Journal of Political Economy, 57,

463-95 (repris in M. Friedman, Essays in Positive Economics, 1953). (1953) * The Methodology of Positive Economics *, in M. Friedman, Essays in Positive Economics. Chicago 1953.

Milton Friedman's Monetary Framework, ouvr. collectif, Chicago.

Hempel, C. G. (1954) * A Logical Appraisal of Operationism \#, Scientific Monthly, 79, 215-20 (repris in Hempel 1965, p. 123-33).

(1958) * The Theoretician's Dilemma *, in H. Feigl, M. Scriven et G. Maxwell, Minnesota Studies in the Philosophy of Science, vol. 2. Minneapolis. (Repris in Hempel 1965, p. 173-226.) (1965) Aspects of Scientific Explanation and Other Essays in the Philosophy of Science. New York.

(1970) * On the 'Standard Conception' of Scientific Theories $»$, in M. Radner et S. Winokur, Minnesota Studies in the Philosophy of Science, vol. 4. Minneapolis.

Hutchison, T. (1938) The Significance and Basic Postulates of Economic Theory, rééd. New York.

Jacob, P. (1987) * Réalisme scientifique et théorie de la vérité \#, Fundamenta Scientiae, 7 , 333-55.

Knight, F. (1921) Risk, Uncertainty and Profit, reprod. London School of Economics, Series of Reprints of Scarce Tracts. London 1957.

Kreisel, G. et J. L. Krivine (1967) Eléments de logique mathématique, Théorie des modèles. Paris.

Machlup, F. (1946) * Marginal Analysis and Empirical Research », American Economic Review, 36, 519-54. (1952) The Political Economy of Monopoly. Baltimore.

Malinvaud, E. (1971) Leçons de théorie micro-économique. Paris.

Marshall, A. (1920) Principles of Economics, 6e éd. London.

Meidinger, C. (1983) * L'approche économique du comportement humain $»$, in $C$. Meidinger (sous la dir. de), La nouvelle économie libérale, chap. 1. Paris.

Mélitz, J. (1965) * Friedman and Machlup on the Significance of Testing Economic Assumptions \#, Journal of Political Economy, 73, 37-60.

Mingat, A., P. Salmon et A. Wolfelsperger (1985) Méthodologie économique. Paris.

Mongin, P. (1986) * La controverse sur l'entreprise (1940-1950) et la formation de l'irréalisme méthodologique , Ecónomies et Sociétés, série Oeconomia, 5, 95-151. (1987) * L'instrumentalisme dans 1'Essai de M. Friedman », Economies et Sociétés, série Oeconomia, 10, 73-106.

Nagel, E. (1961) The Structure of Science: Problems in the Logic of Scientific Explanation. London.

(1963) * Assumptions in Economic Theory *, American Economic Review, 53, Papers and Proceedings, 211-19, repris in A. Ryan, The Philosophy of Social Explanation. Oxford, p. 130-38.

Pariente, J. C. (1973) Le langage et l'individuel. Paris.

Popper, K. R. (1963) Conjectures and Refutations: The Growth of Scientific Knowledge, Se éd. révisée, 1966. London.

Ramsey, F. (1931) * Theories ", repris in F. Ramsey, The Foundations of Mathematics and Other Logical Essays. New York.

Rosenberg, A. (1976) Microeconomic Laws. Pittsburgh.

Rynaciewicz, R. A. (1983) * Falsifiability and the Semantic Eliminability of Theoretical Languages *, British Journal for the Philosophy of Science, 34, 225-41.

Salmon, P. (1976) * La méthode hypothético-déductive et les raisonnements en termes de 'comme si' en économique. Illustration par la théorie moderne du marché financier ${ }^{\prime}$, Revue d'économie politique, 86, 765-94. 
Samuelson, P. A. (1938) * A Note on the Pure Theory of Consumers' Behaviour *, Economica, 5, 61-71, repris in The Collected Economic Papers of P. A. Samuelson, 2 vols. réunis par J. E. Stiglitz. Cambridge, Mass. 1966, vol. 1, p. 3-14. (1947) The Foundations of Economic Analysis. Cambridge. (1963) * Problems of Methodology-Discussion », American Economic Review, 53, Papers and Proceedings, 231-36.

Scheffler, I. (1963) The Anatomy of Inquiry. Indianapolis.

Schoenfield, J. R. (1967) Mathematical Logic. Reading, Mass.

Simon, H. A. (1977) Models of Discovery. Dordrecht. (1983) * Falsifiability and the Semantic Eliminability of Theoretical Languages *, British Journal for the Philosophy of Science, 34, 225-41.

Stegmuiller, W. (1976) The Structure and Dynamics of Theories. New York.

Tuomela, R. (1973) Theoretical Concepts. Vienna.

Vaihinger, H. (1911) Die Philosophie des Als Ob. System der theoretischen, praktischen und religiösen Fiktionen der Menschheit auf Grund eines idealistischen Positivismus. Berlin. 